\title{
On Possibility of Detection of Variable Sources Using the Data of "Cold" Surveys Carried Out on RATAN-600
}

\author{
E. K. Majorova ${ }^{1}$ and O. P. Zhelenkova ${ }^{1}$ \\ ${ }^{1}$ Special Astrophysical Observatory of the Russian AS, Nizhnij Arkhyz 369167, Russia
}

In this study we attempt to assess the possibility of detection of variable sources using the data of the 7.6 -cm wavelength surveys carried out on the RATAN-600 radio telescope in the period from 1980 through 1994. Objects selected according to certain criteria from the RCR catalog are used to construct the calibration curves and to estimate the accuracy of the resulting calibration curves and determine the r.m.s. errors for the measured source flux densities. To check the calibration sources for the presence of variable objects, quantitative estimates are performed for a number of parameters that characterize variability, in particular, for the long-term variability index $V$ and the $\chi^{2}$ (chi-square) probability $p$. The long-term variability index was found to be positive for 14 out of approximately 80 calibration sources, possibly indicating that these sources are variable. The most likely candidate variables are the three sources with the $\chi^{2}$ probability $p>0.95$. Five sources have $\chi^{2}$ probabilities in the $0.85<p<0.95$ interval, and the remaining six in the $0.6<p<0.8$ interval. Nine out of 14 objects are possibly variable in the optical range. The light curves and spectra are determined for possible variable sources and a number of "non-variable" objects. We plan to use the results of this study in our future searches for variable radio sources using the data of the "Cold" surveys.

\section{INTRODUCTION}

The problem of searching for variability of cosmic objects was already formulated during the preparation phase of the first deep search surveys on the RATAN-600 radio telescope, namely the "Cold" [1] and Zelenchuk surveys [2]. The samples of radio sources obtained as a result of the Zelenchuk survey at 3.9 and $7.5 \mathrm{GHz}[3-5]$ formed the basis for the first studies of variable sources on RATAN-600. The results of the analysis of their statistical properties can be found in [6].

Starting from 1998, long-term sets of mul- tifrequency observations have been carried out on the Northern sector of the radio telescope to study variable objects. The duration of continuous daily observations of the same sources ranged from one to three months. These studies targeted mostly discrete bright radio sources with flat spectra. Such sources exhibit variations on time scales ranging from tens of minutes to several decades. The results of these long-term studies were reported in many publications by the researchers from the Sternberg Astronomical Institute, the Special Astrophysical Observatory of the Russian Academy of Sciences, and the Astro 
Space Center of the Lebedev Physical Institute of the Russian Academy of Sciences, e.g. [7-13].

In this paper we analyze the possibility of discovery of variable radio sources based on the data of the deep surveys carried out on the Northern sector of the RATAN-600 radio telescope from 1980 through 1999.

To this end, we use a sample of calibration sources selected by certain criteria to construct the calibration curves and perform detailed estimates of the flux density measurement errors.

We use several criteria, including statistical ones, for quantitative estimates of the possible variability of the objects studied, and construct light curves for suspected variable sources and a number of "non-variable" objects.

\section{DEEP SURVEYS ON RATAN-600}

In 1980 the first $3.94 \mathrm{GHz}$ deep blind survey was performed on the Northern sector of RATAN-600 within the framework of the "Cold" experiment $[1,14]$ at the declination of the SS 433 source. Practically at the same time the multifrequency Zelenchuk survey [3, 4] was carried out with a flat reflector on the Southern sector.

Starting from 1998 the multiwavelength $(\lambda=1-55 \mathrm{~cm})$ RZF zenith survey $[15,16]$ was carried out on the Northern sector. This survey was carried out in 9 and 17 sections since 2001 and 2006, respectively.

A radio-source catalog (the $\mathrm{RC}$ catalog) with a detection threshold of $10 \mathrm{mJy}[17,18]$ was produced based on the data of the "Cold" survey. To refine the flux densities and coordinates of the RC catalog sources, several more observing runs were carried out on the Northern sector of the radio telescope at the same frequency and at the same declination $\left(\operatorname{Dec}_{1980}=4^{\circ} 57^{\prime}\right)$.

The results of the reduction of these observations were reported in $[16,19,20]^{1}$. Soboleva et al. [21] reported the results obtained using newly reduced records of the "Cold-80" experiment in the interval of right ascensions $7^{\mathrm{h}} \leq R A<17^{\mathrm{h}}$. The list of objects found in this strip and identified with the objects of the NVSS catalog [24] can be found in the RCR (RATAN Cold Refined) catalog. ${ }^{2}$

The reduction of the data of these surveys revealed that the flux densities of a number of objects vary from one observing run to another. The authors of the above studies averaged the flux densities over all the observing runs, since identifying variable radio sources was not among their tasks. These averaged flux densities and their errors are reported in the RCR catalog [21].

In this paper we try to analyze whether it is possible to discover variable radio sources in the search surveys.

To solve this problem, we use the data of the 7.6-cm surveys carried out in 1980, 1988, 1993,

\footnotetext{
${ }^{1}$ Bursov [16] gives a complete bibliography of papers published on the subject.

${ }^{2}$ The spectra of the RCR catalog sources are available at http://www.sao.ru/hq/len/RCR/.
} 
and 1994 at the declination of $D e c_{1980}=4^{\circ} 57^{\prime}$ in the $7^{\mathrm{h}} \leq R A<17^{\mathrm{h}}$ strip. The detection thresholds (or the average $\overline{3 \sigma}$ values) in these surveys were equal to $8.0 \pm 0.5 \mathrm{mJy}$ for the 1980 survey; $10.6 \pm 1.3 \mathrm{mJy}$ for the 1988 survey; $10.4 \pm 3.7 \mathrm{mJy}$ for the 1993 survey; $9.6 \pm 1.2 \mathrm{mJy}$ for the 1994 survey; $13.5 \pm 5.5 \mathrm{mJy}$ for the $1994\left(H=51^{\circ} 09^{\prime}\right)$ survey, $11.1 \pm 2.0 \mathrm{mJy}$ for the $\left(H=51^{\circ} 22^{\prime}\right)$ survey $^{3}[21]$ ( $H$ is the elevation to which the antenna was set during the survey).

Here we do not analyze the data of the 1990, 1991, and 1999 surveys carried out at the same wavelength and declination because of their lower sensitivity. We will return to these surveys later.

The use of surveys to study the variability of radio sources has a certain advantage due to the fact that in the process of the survey the antenna is focused onto a certain elevation $H$ (declination $D e c_{0}$ of the central survey section) and its configuration remains practically unchanged during the observations.

This reduces the errors due to the repositioning of the antenna, which is especially important for the determination of flux densities of faint sources. Studies of variable sources carried out in the mode described by Gorshkov et al. [13] involve repeated repositioning of the antenna to different areas of the sky.

Another advantage of search surveys is that due to the specificity of the power beam pat-

\footnotetext{
${ }^{3}$ In 1994 the antenna was set not only to the declination of the SS 433 source, but also to $4^{\prime}$ above $\left(H=51^{\circ} 22^{\prime}\right)$ or below $\left(H=51^{\circ} 09^{\prime}\right)$ this declination.
}

tern (PBP) of RATAN-600 its field simultaneously covers many sources in a single run of the sky strip. ${ }^{4}$

The number of sources crossing the PBP that can be identified in records increases with the sensitivity of the telescope and integration time. Integration time is determined by the number of repeated transits of the given sky strip (i.e., the number of scans).

The number of transits of the observed sky strip in the surveys considered varied from 20 to 35 depending on the survey and hour of observation. In the programs described by Gorshkov et al. [13] each source was observed three to six times.

Thus repeated scanning of the same sky strip in the surveys not only increases the number of objects, but also makes it possible to study fainter sources compared to the mode described by Gorshkov et al. [13].

Note that the data of the considered surveys can be used to study the long-term variability of radio sources on time scales of several years, which is known to be due to the nonstationary processes in active galactic nuclei.

\footnotetext{
${ }^{4}$ More than 30000 radio sources cross the PBP of RATAN-600 through the area within the sheet envelope in a single crossing of the sky at $\lambda 7.6 \mathrm{~cm} \mathrm{[24].}$
} 

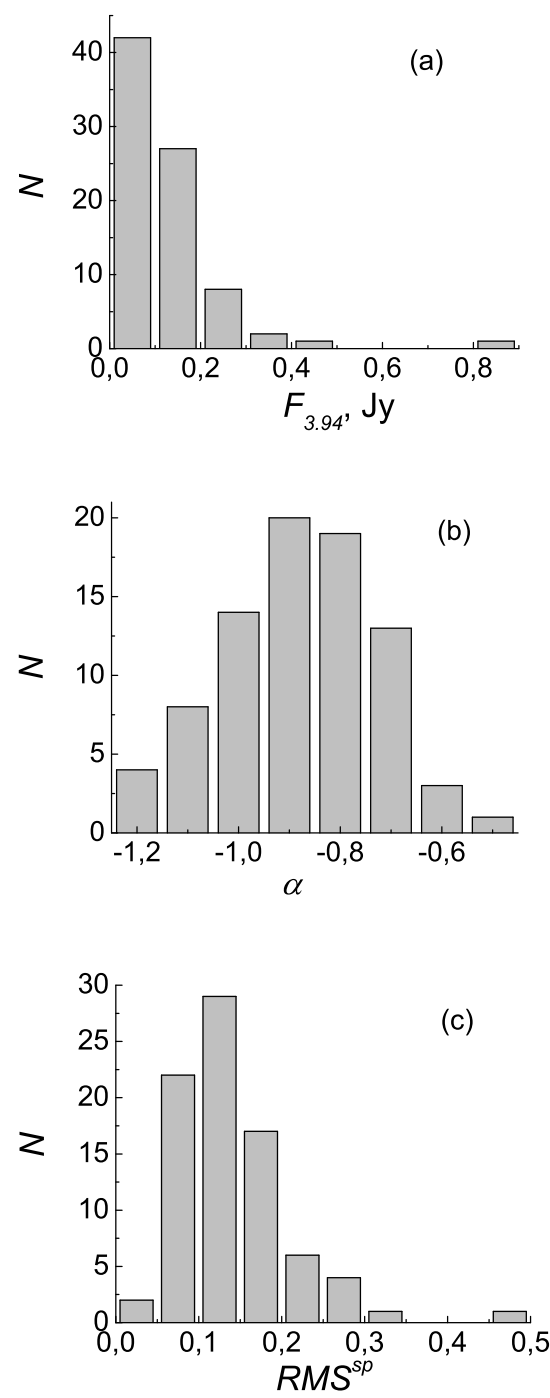

Figure 1. Histograms of the flux densities (a), spectral indices (b), and the r.m.s. error of the scatter of data points in the spectra (c) for calibration sources.

\section{SELECTION OF CALIBRATION SOURCES}

The principal aim of this work is to derive the calibration curves that can be used to compute the source flux densities and to estimate the flux density errors.

To derive these calibration curves, we selected
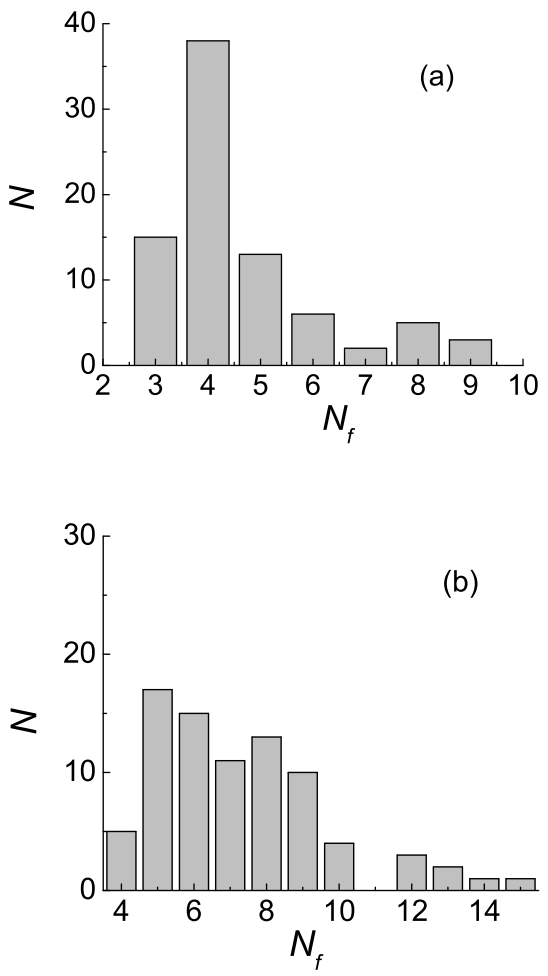

Figure 2. Histograms of the number of frequencies for which the flux density data are available for the spectra of radio sources. The left-hand panel shows the distribution based on the NED data exclusively, and the right-hand panel shows the distribution based on all the available data collected from different catalogs including the data of RATAN-600 surveys, and on the estimates

based on the maps of the VLSS and GB6 surveys.

RCR radio sources with steep and well-studied spectra with available flux density data at several frequencies. We selected sufficiently bright objects with minimal scatter of data points in their spectra.

Radio sources with steep spectra seldom exhibit variations at frequencies greater than $1 \mathrm{GHz}$. However, such variability is observed in objects where a compact component is found, which is responsible for flux density varia- 
tions [22, 23]. Our sample does not include known variable sources, which have mostly flat spectra.

We selected a total of sources with flux densities $F_{3.94}>$ 40 mJy and six more sources with $F_{3.94} \sim 30$ mJy. $\quad\left(F_{3.94}\right.$ is the flux density at $3.94 \mathrm{GHz}$.) Note that the number of calibration sources somewhat changed from one survey to another.

Figure 1 shows the histograms of the following properties of calibration sources: flux densities $F_{3.94}(\mathrm{a})$, spectral indices $\alpha(\mathrm{b})$, and the relative r.m.s. scatter of data points, $R M S^{s p}$, on their spectra (c).

The r.m.s. (root mean square) error $R M S^{s p}$ of the scatter of data points on the spectrum relative to the approximating curve is normalized to the $3.94 \mathrm{GHz}$ flux density of the source. We fitted the approximating curve (or parabola) using the least squares method.

Most of the selected sources have spectral indices $^{5} \alpha_{3.94}<-0.75\left(F_{f} \sim f^{\alpha}\right)$ and r.m.s. errors of the scatter of data points on the spectrum $R M S^{s p}<20 \%$.

The average $R M S^{s p}$ value for the entire sample of calibration sources was $\overline{R M S^{s p}}=0.12 \pm 0.06$. According to the data of the used catalogs, the source flux density errors at different frequencies lie in the interval from $6 \%$ to $28 \%$. The average flux density error

\footnotetext{
${ }^{5} \alpha_{3.94}$ is the spectral index at $f=3.94 \mathrm{GHz}$.
}

for the entire sample of calibration sources is $15 \% \pm 0.03 \%$.

Most of the calibration sources appear double on the FIRST radio maps, and a minor fraction of them are point sources, identified both with galaxies and quasars.

Figure 2 shows the histograms of the number of frequencies for which the data on the flux densities in the spectra of radio sources are available.

The histogram in the left panel takes into account only the data available in the NED database [25], and that in the right panel, all the available data collected from various catalogs including the RATAN-600 surveys and our estimates [21] based on VLSS maps [26] and GB6 surveys [27]. It is evident from the histograms that the NED data for the selected calibration sources are available at four or more frequencies, and with other catalogs taken into account, the data coverage increases to include five to nine frequencies for the overwhelming majority of the sources.

\section{CONSTRUCTION OF THE} CALIBRATING CURVES AND

\section{ESTIMATION OF THE SOURCE FLUX DENSITY ERRORS}

Let us recall some of the features of the observations on the RATAN-600 radio telescope whose PBP differs significantly from that of a parabolic dish [28-32]. In the mode of single-sector observations the $\mathrm{PBP}$ broadens with increasing angu- 

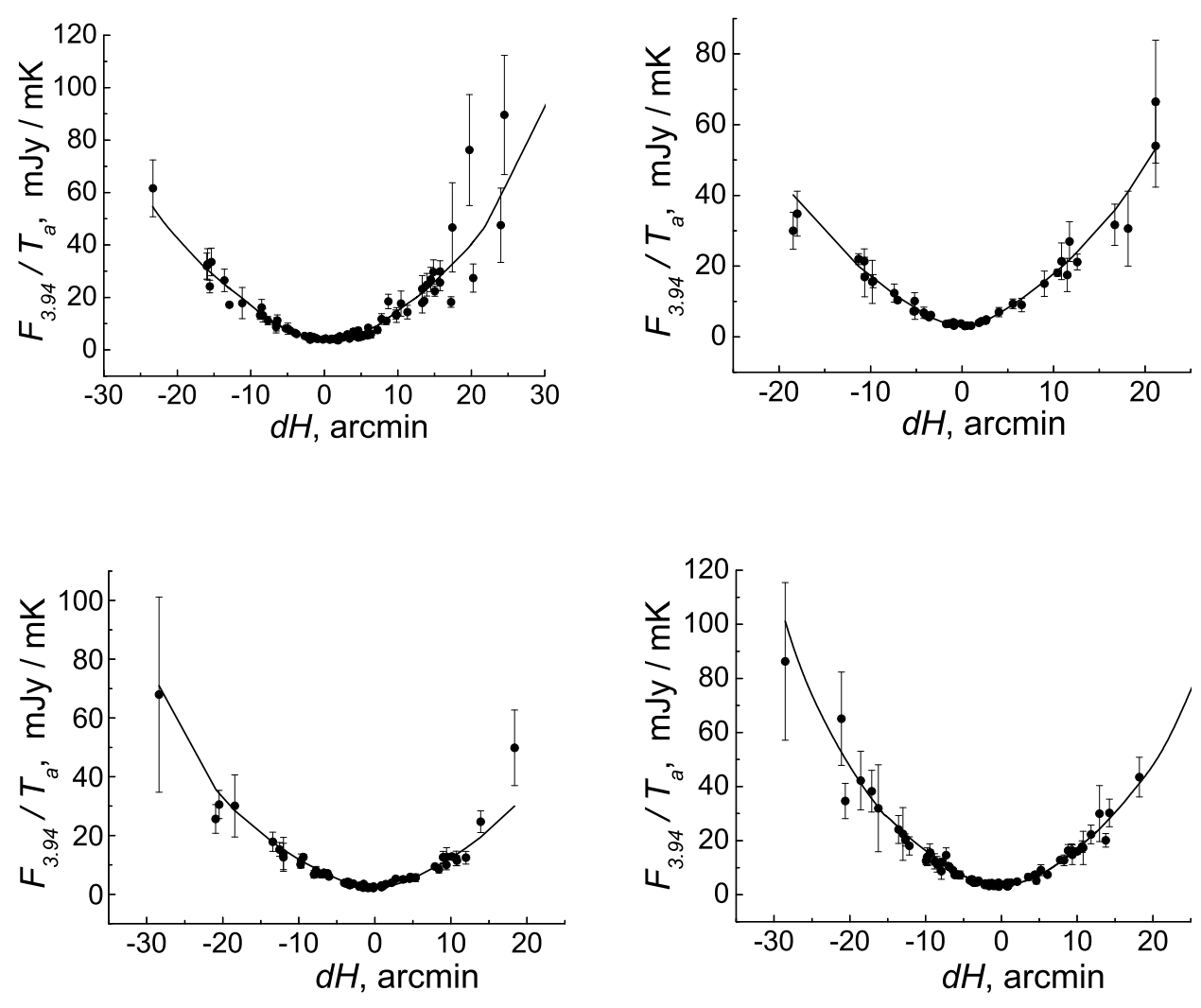

Figure 3. Dependence of the $F_{3.94} / T_{a}$ ratio on $d H$ (circles) based on the data for the calibration sources from the 1980, 1988, 1993, and 1994 surveys (from left to right and from top to bottom) and the computed $A / k_{P B P}(d H)$ curves (the solid lines).

lar distance from its central section. Correspondingly, the farther the source is from the central section, the broader is the response width and the weaker the signal.

One-dimensional scans are superpositions of the sources that have crossed different horizontal sections of the power beam pattern.

We repeated the reduction of the selected sources. Our initial data consisted of the averaged records of several-day long observations that have already been subjected to primary re- duction [16]. After background subtraction ${ }^{6}$ the sources were identified on the averaged scans using the Gaussian analysis. We performed the entire procedure using the standard software for the reduction of radio astronomical observations [33].

The temporal calibration was based on the strong sources, with the use of the data from the NVSS catalog. For each source identified in the record we determined its antenna temperature $T_{a}^{i}$, halfwidth $H P B W^{i}$ of the Gaussian fit, and

\footnotetext{
${ }^{6}$ The background was computed with an 80-s "smoothing window" to prevent suppression of the signal from the sources located far from the central section [21].
} 
the right ascension $R A^{i}$.

In our analysis of the data we used the information on the declination offset $d H$ of the source relative to the central section of the survey and the computed [31] $H P B W(d H)$ dependences, where $H P B W$ is the halfwidth of the vertical PBP, $d H=\Delta D e c=D e c^{i}-D e c^{0}, \quad D e c^{i} \quad$ is the declination of the $i$-th source, and $D e c^{0}$ is the declination of the central section of the survey. A comparison of the source halfwidths $H P B W^{i}$, determined from the Gaussian analysis, and the $H P B W(d H)$ dependences tested experimentally by Majorova and Trushkin [32] and Majorova and Bursov [34], allowed us to control the reliability of the extraction of these objects.

We then constructed for each survey the dependences of $F_{3.94}{ }^{i} / T_{a}{ }^{i}$ on $d H$. Here $F_{3.94}{ }^{i}$ is the 3.94-GHz flux density of the calibration source and $T_{a}{ }^{i}$ is its antenna temperature. We determined $F_{3.94}{ }^{i}$ from the approximating curve of the spectrum of the corresponding source, and $T_{a}{ }^{i}$ from the Gaussian analysis of the averaged survey record. The circles in Fig. 3 show the $F_{3.94}{ }^{i} / T_{a}^{i}$ ratios based on the data of the 1980, 1988, 1993, and 1994 surveys (from left to right and from top to bottom). The solid lines show the computed calibrating curves $A / k_{P B P}(d H)$, where $k_{P B P}(d H)$ is the pattern factor. It is equal to the vertical PBP $F_{v}$ of the telescope if the primary feed is located at the focus of the antenna, or to the dependence of the maximum value of the PBP at different horizontal sections on the offset of this section relative to the central section in the case of nonzero transversal off-focus offset of the feed.

We computed the pattern factor $k_{P B P}(d H)$ for each survey using the algorithms described by Majorova [31]. Its value indicates to what extent the response to the source weakens with increasing distance from the central section of the survey (or the central section of the PBP).

We computed the $k_{P B P}(d H)$ taking into account the transversal offset of the primary feed (horn). The greatest off-focus offset of the horn was used during the "Cold" survey in 1980, and in 1988 the horn was located at the focus of the antenna.

Unlike Soboleva et al. [21] and Majorova [35], in this paper we compute the pattern factor $k_{P B P}(d H)$ for the 1980 survey taking into account both the transversal offset of the horn and the horn offset along the direction making an angle of $50^{\circ}$ to the horizon and also a small longitudinal offset. Additional off-focus offsets were applied in the process of the "Cold" experiment in order to reduce the noise temperature of the antenna. Taking these offsets into account in our computations of the $k_{P B P}(d H)$ factor allowed us to match the computed and experimental data and, in particular, reveal the roughly $1^{\prime}$ offset of the experimental vertical PBP from the corresponding computed one [35]. 
For each survey we chose the $A$ factor $^{7}$ that minimized the standard error $R M S^{k}$ of the scatter of experimental data points $F_{3.94}{ }^{i} / T_{a}{ }^{i}$ relative to the computed calibrating curve $A / k_{P B P}(d H)$.

$R M S^{k}=\sqrt{\frac{1}{N} \sum_{i}^{N}\left(\frac{F_{3.94^{i}} / T_{a}^{i}-A / k_{P B P}}{A / k_{P B P}}\right)^{2}}$,

where $N$ is the number of sources used to construct the calibrating curve for the survey considered.

In this study we somewhat deviated from the technique used by Bursov [19], Soboleva et al. [21], and Majorova and Bursov [34]. In those papers the calibrating curves are the curves fitted to the experimental $F_{3.94}{ }^{i} / T_{a}{ }^{i}$ data points using the least squares method.

Majorova [35] showed that the experimental vertical $\operatorname{PBPs} F_{v}(d H)=k_{P B P}(d H)=$ $A / F_{3.94}{ }^{i} / T_{a}{ }^{i}$ of the radio telescope based on the data of the 1980-1999 surveys agree well with the computed PBPs. We therefore used the $A / k_{P B P}$ ratio as the calibration curve. We computed the $k_{P B P}$ factor taking into account the observing conditions and chose the $A$ factor that would minimize the $R M S^{k}$. We found that in this case the $R M S^{k}$ error averaged over the entire range of $d H$ is smaller than the error of the scatter of experimental data points relative to the leastsquares fitted curve (a second- or fourth-order polynomial).

\footnotetext{
${ }^{7}$ The $A$ factor is equal to the $A=2 k / S_{\text {eff }}$ ratio, where $k$ is the Boltzmann constant and $S_{\text {eff }}$ is the effective area of the radio telescope.
}

A comparison of the calibrating curves derived in this study for the 1988 survey and those by Bursov [19] shows that they practically coincide in the $-10^{\prime}<d H<10^{\prime}$ interval. The curves diverge at greater absolute values of $d H$ and at $d H \sim 20^{\prime}$ the $F_{3.94}{ }^{i} / T_{a}{ }^{i}$ ratios of Bursov [19] exceed our estimates by a factor of 1.4 .

This may be due both to the set of calibration sources and to the adopted reduction technique, in particular, the background computation and its subtraction. The latter factor is especially critical for estimates of the parameters of the sources located far from the central section. Subtraction of the background computed with a "smoothing window" of about $20 \mathrm{~s}$ results in underestimated antenna temperatures for distant sources and, consequently, in the increase of the $F_{3.94}{ }^{i} / T_{a}^{i}$ ratio with increasing of $d H$.

There was yet another reason why we used the computed $A / k_{P B P}(d H)$ dependences instead of the approximating curves: it was done to avoid the influence of variable sources, which may happen to be among the calibration sources.

Table 1 lists the average relative standard errors $R M S^{k}\left(\overline{R M S^{k}}\right)$ computed for the 1980 , 1988, 1993, and 1994 surveys. We performed averaging over the entire range of the $d H$ angles considered and the intervals $-15^{\prime}<d H<15^{\prime}$, $-10^{\prime}<d H<10^{\prime}$, and $-5^{\prime}<d H<5^{\prime}$. The $\overline{R M S^{k}}$ values averaged over the sample of sources whose recorded antenna temperatures exceed $10 \sigma_{s}$ are also listed in the table.

Note that the $\overline{R M S^{k}}$ values for the entire 
range of the $d H$ angles are close to the standard error of the scatter of data points of the experimental PBP relative to the computed PBP obtained by Majorova [35] for a sample of sources with flux densities $F_{3.94}>50 \mathrm{mJy}$. The $\overline{R M S^{k}}$ value for the 1980 survey is smaller than the estimate reported by Majorova [35], which can be explained by the allowance for additional off-focus offsets of the horn in the computation of the pattern factor $k_{P B P}(d H)$.

We estimated the relative standard errors of the $F_{3.94}{ }^{i} / T_{a}{ }^{i}$ ratio and its confidence intervals (Fig. 3) using the relative standard errors of the scatter of data points, $R M S^{s p}$, in the spectra of sources and the relative standard errors $R M S^{T a}=\sigma_{s} / T_{a}{ }^{i}$ of the inferred antenna temperatures, where $\sigma_{s}$ is the dispersion of noise in the sky strip transit records in the considered survey.

Note that if the standard errors $R M S^{s p}$ of the scatter of data points are sufficiently uniformly distributed with respect to angle $d H$ then the errors $R M S^{T a}$ of the antenna temperatures depend significantly on the distance of the source from the central section of the survey. We illustrate this point in Fig. 4, where we show the dependences of $R M S^{T a}$ on $d H$ (a) and $R M S^{s p}$ on $d H$ (b) based on the sample of calibration sources observed in the 1980 survey. The relative standard errors of the $F_{3.94}{ }^{i} / T_{a}{ }^{i}$ ratio $\left(R M S^{F T a}\right)$ also increase with increasing of the angle $d H$ absolute value (Fig. 4c). Table 2 lists the averaged relative standard errors of the $F_{3.94}{ }^{i} / T_{a}{ }^{i}$ ratio.
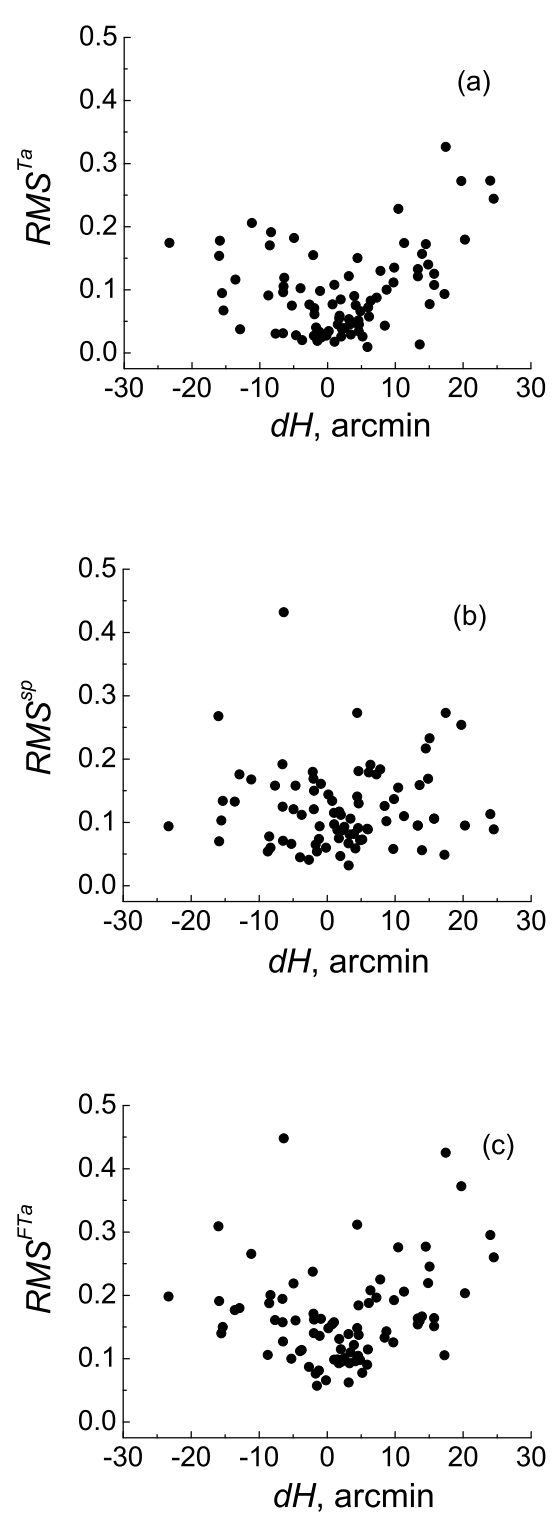

Figure 4. The $R M S^{T a}(d H)$ (a), $R M S^{\mathrm{sp}}(d H)$ (b), and $R M S^{F T a}(d H)$ (c) dependences for the calibration sources based on the data of the 1980 survey.

We averaged these standard errors over the $d H$ intervals indicated in the first column of the table.

A comparison of the data listed in Tables 1 and 2 shows that the errors of the scatter of experimental $F_{3.94}{ }^{i} / T_{a}{ }^{i}$ data points relative to 
Table 1. Averaged relative standard errors $R M S^{k}$ $\left(\overline{R M S^{k}}\right)$

\begin{tabular}{c|l|l|l|l}
\hline & 1980 & 1988 & 1993 & 1994 \\
\hline$-30^{\prime}<d H<30^{\prime}$ & 0.197 & 0.123 & 0.175 & 0.139 \\
$-15^{\prime}<d H<15^{\prime}$ & 0.139 & 0.107 & 0.154 & 0.132 \\
$-10^{\prime}<d H<10^{\prime}$ & 0.141 & 0.100 & 0.153 & 0.132 \\
$-5^{\prime}<d H<5^{\prime}$ & 0.109 & 0.078 & 0.164 & 0.135 \\
$T_{a}>10 \sigma$ & 0.127 & 0.096 & 0.165 & 0.127 \\
\hline
\end{tabular}

Table 2. Averaged relative standard errors of the $F_{i} / T_{a i}\left(\overline{R M S^{F T a}}\right)$ ratio

\begin{tabular}{c|c|c|c|c}
\hline & 1980 & 1988 & 1993 & 1994 \\
\hline$-30^{\prime}<d H<30^{\prime}$ & 0.165 & 0.186 & 0.181 & 0.187 \\
$-15^{\prime}<d H<15^{\prime}$ & 0.156 & 0.178 & 0.171 & 0.181 \\
$-10^{\prime}<d H<10^{\prime}$ & 0.142 & 0.170 & 0.156 & 0.167 \\
$-5^{\prime}<d H<5^{\prime}$ & 0.128 & 0.132 & 0.144 & 0.145 \\
$T_{a}>10 \sigma$ & 0.128 & 0.131 & 0.139 & 0.131 \\
\hline
\end{tabular}

the $A / k_{P B P}(d H)\left(\overline{R M S^{k}}\right)$ calibrating curve are smaller than or comparable to the averaged relative standard errors of the $F_{3.94}{ }^{i} / T_{a}{ }^{i}$ ratio. The only exceptions were the $\overline{R M S^{k}}$ errors for the 1980 survey averaged over the entire $d H$ interval and the $\overline{R M S^{k}}$ errors for the 1993 survey averaged over the $-5^{\prime}<d H<5^{\prime}$ interval. In the case of the 1980 survey the exclusion of the sole source with the largest deviation from the computed curve, J 103938+051031, reduces the $\overline{R M S^{k}}$ to 0.168 , which is comparable to the $\overline{R M S^{F T a}}$ value in the $-30^{\prime}<d H<30^{\prime}$ interval.

Our analysis of the dependences shown in Fig. 3 and the data listed in Table 1 lead us to conclude that the $F_{3.94}{ }^{i} / T_{a}{ }^{i}$ ratios of most of the considered calibration sources are close to the $A / k_{P B P}$ values for the corresponding $d H$ angles and the difference between the two quantities is within the confidence interval of the $F_{3.94}{ }^{i} / T_{a}{ }^{i}$ ratio. These sources mostly lie in the $d H= \pm 15^{\prime}- \pm 17^{\prime}$ interval.

At greater distances of the sources from the central section of the survey $\left(|d H|>17^{\prime}\right)$ deviations of the experimental data points from the $A / k_{P B P}$ curve increase and so do the errors of the $F_{3.94}{ }^{i} / T_{a}{ }^{i}$ ratio. This effect is most conspicuous in the 1980 survey. These deviations may be due to both the accuracy of identification in records of the sources located far from the central section, and to the pattern effects. Neither can we rule out the possibility that our sample may contain variable objects.

The averaged standard errors of the scatter of experimental data points relative to the computed $\overline{R M S^{k}}$ curve are minimal in the 1988 (for $-15^{\prime}<d H<15^{\prime}$ ) and 1980 (for $-5^{\prime}<d H<5^{\prime}$ ) surveys: they are equal to $8 \%$ and $11 \%$, respectively. In the $-15^{\prime}<d H<15^{\prime}$ interval the $\overline{R M S^{k}}$ errors for the 1980, 1993, and 1994 surveys are equal approximately to $14 \%, 15 \%$, and $13 \%$.

In conclusion, we show in Fig. 5 the dependences of the $G=\left(F_{3.94}{ }^{i} / T_{a}^{i}\right) /\left(A / k_{P B P}\right)$ ratio on $d H$ (from left to right and from top to bottom for the 1980, 1988, 1993, and 1994 surveys, respectively). The horizontal lines correspond to $G=1 \pm 3 \overline{R M S^{k}}$

It is evident from these plots that the deviations of the experimental data points from the 
computed curves do not exceed $3 \overline{R M S^{k}}$ except for three points in the 1980 survey and two points in the 1993 survey. They are sufficiently uniformly distributed over the entire range of variation of the $d H$ angles. As we pointed out above, the smallest deviations of the experimental data points from the computed curves are found in the 1988 survey.

The sources whose $F_{3.94}{ }^{i} / T_{a}{ }^{i}$ ratios deviate by more than $\pm 3 \overline{R M S^{k}}$ from the computed curve may be variable. These sources are J 103938+051031, J 110246+045916， J 114220+045459， J 121852+ 051447, and J 142104+050843.

\section{ANALYSIS OF THE SAMPLE OF} CALIBRATION SOURCES FOR THE PRESENCE OF VARIABLE SOURCES

While selecting sources with steep spectra for the construction of the calibrating curves we tried to reduce the likelihood of contamination of our sample by variable sources. However, we cannot completely rule out the presence of such objects in our list.

To test our calibration sources for variability, we performed a number of quantitative estimates and, in particular, estimated the coefficients $V_{R}$ [36], $V_{F}$ [37], and the long-term variability index $V$ [13].

We computed the coefficients using the following formulas:

$$
V_{R}=F_{i} / F_{j}
$$

$$
\begin{gathered}
V_{F}=\frac{F_{i}-F j}{\sqrt{\left(\sigma_{i}^{2}+\sigma_{j}^{2}\right)}}, \\
V=\frac{\left(F_{i}-\sigma_{i}\right)-\left(F_{j}+\sigma_{j}\right)}{\left(F_{i}-\sigma_{i}\right)+\left(F_{j}+\sigma_{j}\right)},
\end{gathered}
$$

where $F_{i}$ and $F_{j}$ are the flux densities of a given source measured in cycle $i$ and $j$ surveys, respectively, and $\sigma_{i}$ and $\sigma_{j}$ are the absolute standard errors of the inferred flux densities $(i, j=80,88,93,94)$.

The latter two criteria take into account the flux density errors, and they can therefore be considered to be more reliable for testing sources for variability.

We computed the flux densities using the following formula:

$$
F=\frac{A}{k_{P B P}} T_{a} .
$$

Here we used the antenna temperatures of the sources $T_{a}$ determined from the averaged records of the $i$-th year survey and the corresponding computed $A / k_{P B P}(d H)$ curves.

We computed the absolute $\left(\sigma_{i}\right)$ and relative $\left(R M S_{i}\right)$ standard errors of the determination of the source flux density in the $i$-th survey using the following formulas:

$$
\begin{gathered}
R M S_{i}=\sqrt{\left(R M S^{k}\right)^{2}+\left(R M S^{T a}\right)^{2}} \\
\sigma_{i}=F_{i} R M S_{i} .
\end{gathered}
$$

We computed the $V_{R}, V_{F}$, and $V$ coefficients for all the calibration sources whose flux densities 

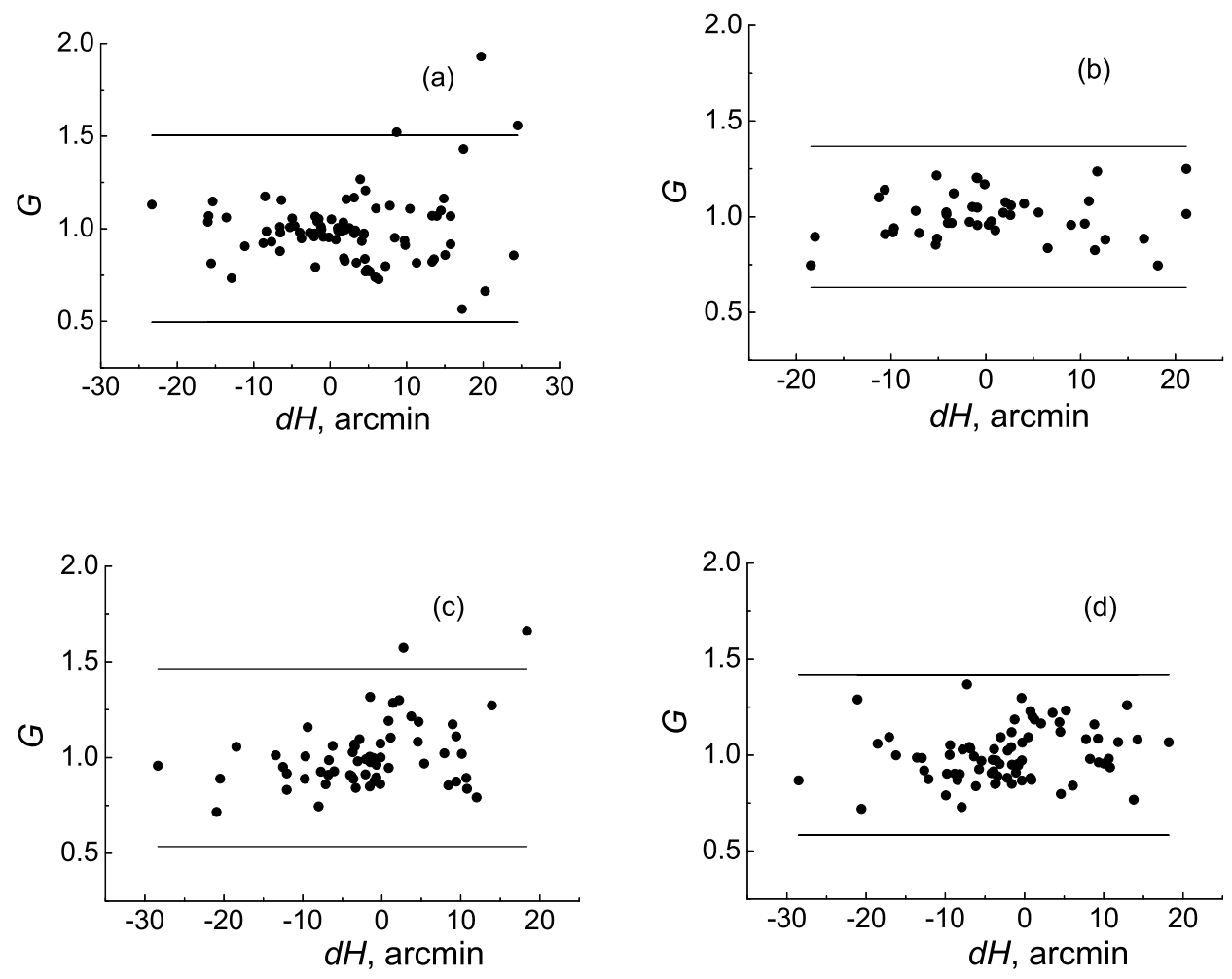

Figure 5. Dependences of the $G=\left(F_{i} / T_{a i}\right) /\left(A / k_{P B P}\right)$ ratio on $d H$ according to the data of the 1980, 1988, 1993, and 1994 surveys (from left to right and from top to bottom). The horizontal lines correspond to $G=1 . \pm 3 \overline{R M S^{k}}$.

are determined in at least three surveys. In our value (column 6).

computations we used the standard errors $R M S^{k}$

listed in Table 1. We suspected a source to be

$\bar{F}=\frac{1}{n} \sum_{i}^{n} F_{i}$,

variable if it had a positive long-term variability

index $(V>0)$. For such sources the flux density difference determined in different surveys differed by more than the sum of standard errors in these surveys.

In the entire sample of calibration sources, 14 objects had a positive $V$ index for at least one pair of surveys. Table 3 lists the coefficients $V, V_{R}$, and $V_{F}$ for these objects (columns 2, 3, considered (columns 6 and 7). Let us recall that and 4), their average flux densities $\bar{F}$ (column 5), due to precession, the source declinations and $d H$ and the standard deviations $\sigma^{\text {set }}$ from the mean varied from one survey to another. The last col- 
Table 3. The $V, V_{F}$, and $V_{R}$ coefficients

\begin{tabular}{|c|c|c|c|c|c|c|c|c|c|}
\hline $\begin{array}{c}R A_{2000} \quad D E C_{2000} \\
\mathrm{RCR} \\
(1)\end{array}$ & $(2)$ & $V_{F}$ & $V_{R}$ & $\begin{array}{c}\bar{F} \\
\mathrm{mJy} \\
(5)\end{array}$ & $\begin{array}{c}\sigma^{s e t}, \\
\text { mJy } \\
(6)\end{array}$ & $R M S^{\text {set }}$ & $\begin{array}{c}d H_{1} \\
\operatorname{arcmin} \\
(8)\end{array}$ & $\begin{array}{c}d H_{2} \\
\operatorname{arcmin} \\
(9)\end{array}$ & $(10)$ \\
\hline J $103938.62+05$ & .264 & 3.13 & 2.53 & 184 & 70 & 0.381 & 19.74 & 13.78 & -0.68 \\
\hline $\mathrm{J} 155148.09+045930.5$ & 0.125 & 2.53 & 1.75 & 79 & 25 & 0.312 & 6.13 & 2.20 & -1.17 \\
\hline $\mathrm{J} 142104.21+050845.0$ & 0.092 & 2.33 & 1.79 & 183 & 53 & 0.293 & 17.25 & 11.83 & -0.79 \\
\hline $\mathrm{J} 132448.14+04$ & 088 & 1.85 & 1.50 & 65 & 13 & 0.233 & 7.23 & 1.26 & -1.03 \\
\hline J $135137.56+043542.0$ & 0.084 & 2.13 & 1.59 & 392 & 99 & 0.262 & -15.36 & -18.46 & -0.89 \\
\hline $\mathrm{J} 11$ & 0.082 & 2.18 & 1.57 & 102 & 26 & 0.260 & 5.56 & 2.75 & -0.81 \\
\hline J $074239.34+050704.3$ & 0.077 & 2.17 & 1.56 & 350 & 74 & 0.211 & -9.40 & -12.90 & -0.85 \\
\hline J $121328.89+050009.9$ & 0.076 & 1.76 & 1.51 & 72 & 15 & 0.213 & 6.52 & -0.39 & -1.07 \\
\hline J 101515.53+045305.6 & 0.061 & 2.16 & 1.41 & 124 & 18 & 0.147 & -0.88 & -3.73 & -1.04 \\
\hline $\mathrm{J} 112437.45+045618.8$ & 0.057 & 1.95 & 1.45 & 466 & 84 & 0.180 & 5.88 & -0.17 & -0.87 \\
\hline J $104551.72+045552.9$ & 0.035 & 1.51 & 1.31 & 157 & 17 & 0.111 & 5.17 & 2.09 & -0.99 \\
\hline J $134243.57+050431.5$ & 0.008 & 1.70 & 1.35 & 973 & 134 & 0.138 & 13.58 & 7.76 & -0.72 \\
\hline J $140730.77+044934.9$ & 0.007 & 1.53 & 1.55 & 82 & 18 & 0.216 & -7.12 & -7.28 & -0.75 \\
\hline J $121852.16+051449.4$ & 0.007 & 1.48 & 1.72 & 237 & 60 & 0.267 & 21.17 & 18.38 & -0.67 \\
\hline
\end{tabular}

umn of Table 3 lists the spectral indices of these objects at $3.94 \mathrm{GHz}$.

Figure 6 shows the dependences of the variability index $V$ on angle $d H$ (a) and the dependences $V_{R}(d H)(\mathrm{b})$ and $V_{F}(d H)$ (c) for the sources from Table 3. We discuss the $V_{\chi}(d H)$ dependence shown in Fig. 6d in Section 6. As is evident from these plots, the radio sources are distributed rather uniformly relative to the central section of the survey. They are also uniformly distributed over the observing hours (or by integrating about 25 transits. in right ascension).

Ten sources with positive long-term variabil- also estimated the long-term variability index ity indices have the coefficients $V_{R}>1.5$, and $V$, eliminating a small systematic trend in four sources have the $V_{R}$ coefficients in the the scatter of the data points of the 1993 

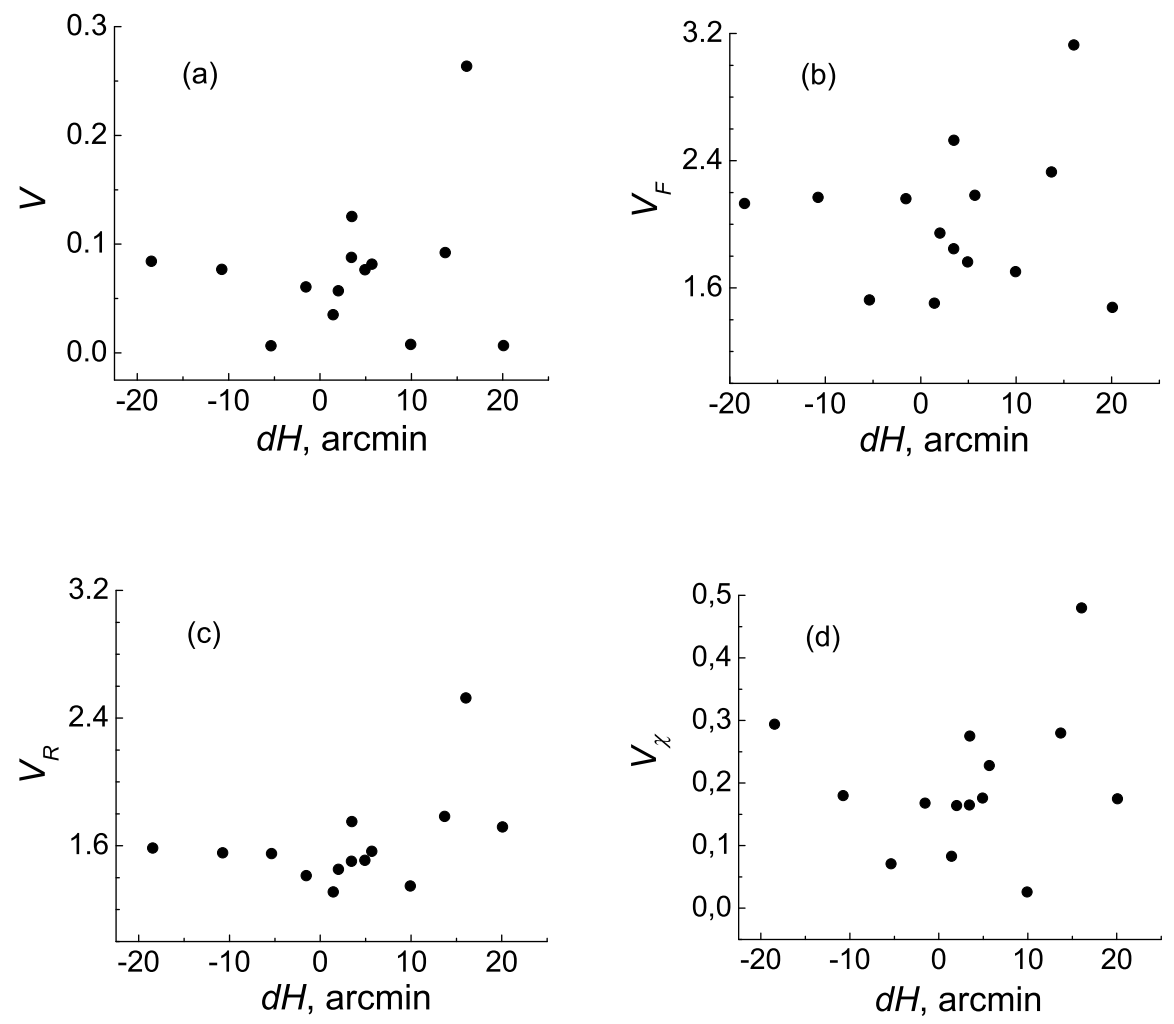

Figure 6. Dependences of the variability indices $V$ (a), $V_{R}$ (b), $V_{F}$ (c), and $V_{\chi}$ (d) on angle $d H$ for 14 suspected variable sources (Tables 3 and 4 ).

and 1994 surveys (Fig. 5). As a result, the the relative standard error of the scatter of data $V$ indices became negative for three out of points in the spectrum of the source or the er14 sources. These objects-J $121852+051449$, ror of determination of its flux density from the $\mathrm{J} 134243+050431$, and $\mathrm{J} 140730+044934$ - prove approximating curve fitted to its spectrum, and to be the least likely variable source candidates. $R M S^{F}$ is the standard error of the flux density

Let us now return to the accuracy of flux density determination for the calibration sources and compare the relative standard errors $R M S^{s e t}$, $R M S^{s p}$, and $R M S^{F}$ for two subsamples. One of these subsamples includes 14 suspected variable sources (Table 3), and the other one includes the "non-variable" sources with $V<0$.

Here $R M S^{\text {set }}$ is the relative standard deviation from the mean flux density $\bar{F}, R M S^{s p}$ is averaged over all surveys.

$$
\begin{gathered}
R M S^{\text {set }}=\sigma^{\text {set }} \bar{F}, \\
R M S^{F}=\frac{1}{n} \sum_{i}^{n} R M S_{i} .
\end{gathered}
$$

Here $R M S_{i}$ is the relative standard error of the flux density of the source in the $i$-th survey, computed using formula (5).

Figure 7 shows the $R M S^{s p}$ (a), $R M S^{F}$ (b), and $R M S^{s e t}$ (c) quantities for the subsample of 

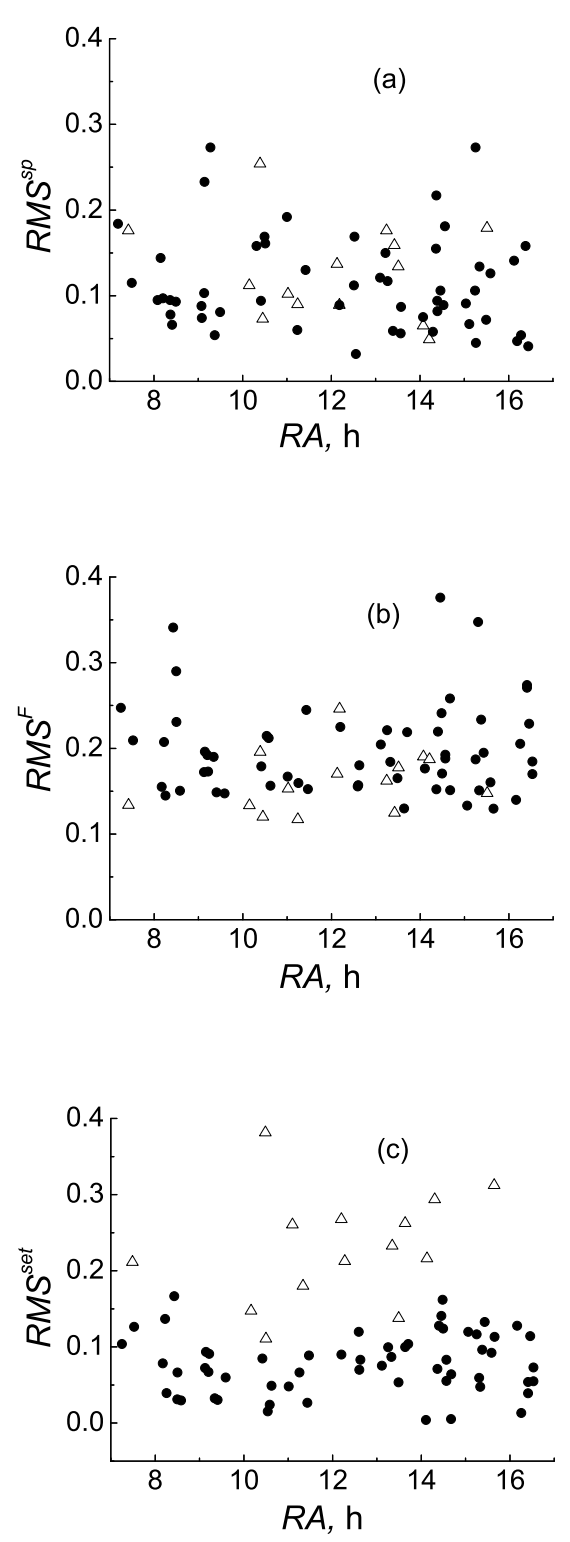

Figure 7. Dependences of the standard errors $R M S^{s p}(R A)(\mathrm{a}), R M S^{F}(R A)(\mathrm{b})$, and $R M S^{\text {set }}(R A)$

(c) for the subsample of 14 suspected variable sources (open triangles) and for the subsample of "non-variable" objects (filled circles).

suspected variable radio sources (open triangles) and for the subsample of "non-variable" objects (filled circles) as a function of $R A$.

It is evident from the above dependences that the standard errors $R M S^{s p}$ of the source flux density inferred from the spectral curves and the standard errors $R M S^{F}$ averaged over all the surveys practically do not differ for the two subsamples of the calibration sources.

As for the standard deviations $R M S^{\text {set }}$, their values for the first subsample consisting of sources with $V>0$ exceed significantly the $R M S^{\text {set }}$ values for the second subsample $(V<0)$. Ten out of 14 candidate variable sources have $R M S^{\text {set }}>0.2$. The average $\overline{R M S^{s e t}}$ for the first and second subsamples of objects are equal to $0.23 \pm 0.07$ and $0.08 \pm 0.04$, respectively.

We compare in Figs. 8 and 9 the $R M S^{\text {set }}$ values with $R M S^{s p}$ and $R M S^{F}$. The filled circles show the $R M S^{s e t}(d H)$ dependences, and the open circles-the $R M S^{s p}(d H)$ (Fig. 8) and $R M S^{F}(d H)$ (Fig. 9) dependences, respectively. Panels (a) and (b) show the corresponding dependences for the "non-variable" sources and the sources listed in Table 3, respectively.

The relative standard deviations $R M S^{\text {set }}$ for the "non-variable" sources are comparable to the relative standard errors $R M S^{s p}$ of the determination of flux densities from the spectra and are substantially smaller than the mean standard errors $R M S^{F}$ of the inferred flux densities averaged over all the surveys.

The $R M S^{\text {set }}$ values for objects with positive long-term variability indices exceed both the $R M S^{F}$ and the $R M S^{s p}$; in the latter case, by almost a factor of two, on average. 

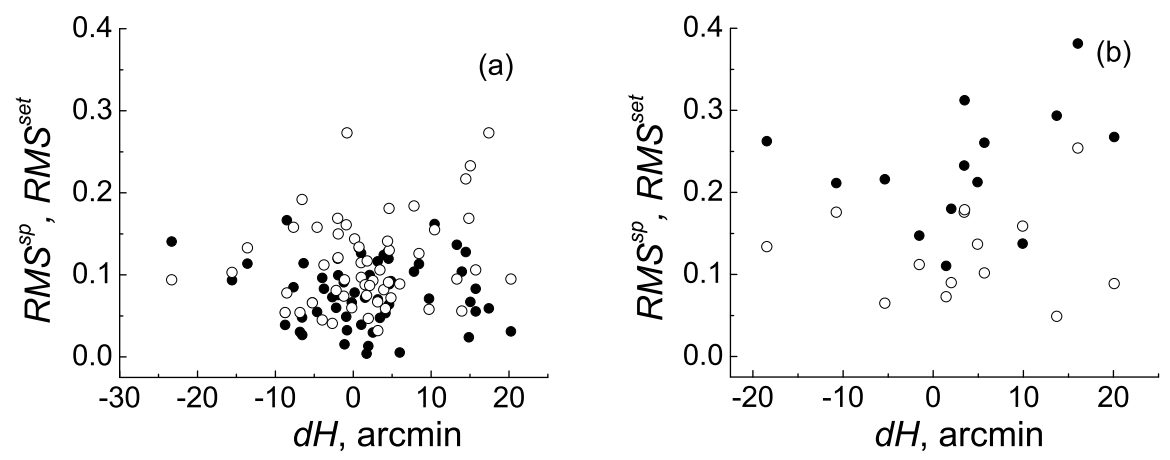

Figure 8. Dependences of the standard errors $R M S^{\text {set }}(d H)$ (filled circles) and $R M S^{\text {sp }}(d H)$ (open circles) for "non-variable" (a) and suspected variable (b) sources.
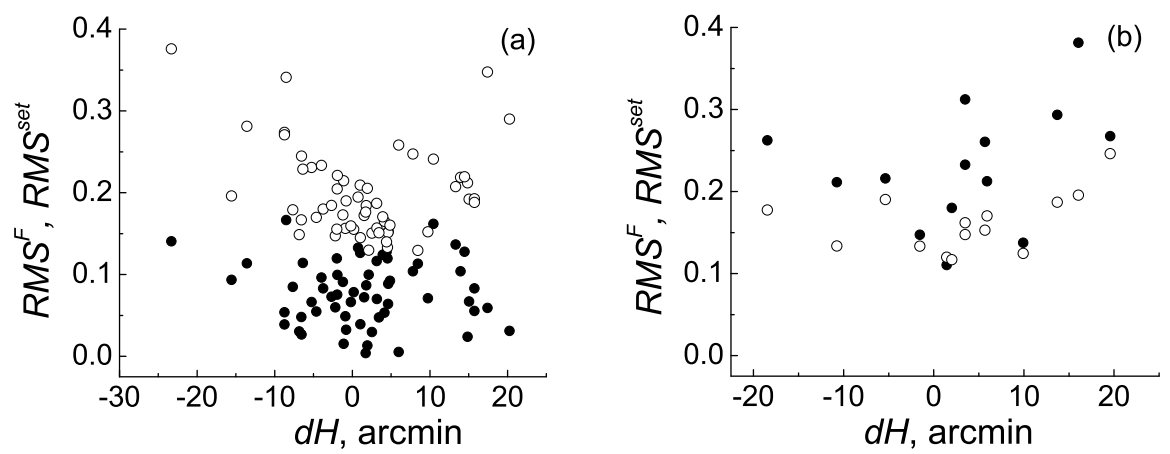

Figure 9. Dependences of the standard errors $R M S^{\text {set }}(d H)$ (filled circles) and $R M S^{\mathrm{F}}(d H)$ (open circles) for "non-variable" (a) and suspected variable (b) sources.

6. ANALYSIS OF STATISTICAL PROPERTIES OF SUSPECTED

\section{VARIABLE SOURCES}

Let us now use statistical techniques to estimate the variability of the sources listed in Table 3, although, unfortunately, the number of data points is rather small for such an analysis.

To confirm the variability of the objects with positive $V$, we performed computations similar to those made by Gorshkov and Konnikova [6], Kesteven et al. [39], Fanti et al. [40], and Seiel- stad et al. [38].

We computed for each of the $n$ surveys the variability amplitude $\Delta F$ and the parameter $V_{\chi}$, as well as the weighted average source flux density $\langle F\rangle$, the weighted average standard error $\langle\sigma\rangle$, and the $\chi^{2}$ constant for $d f=n-1$ degrees of freedom. We computed these quantities using the following formulas [38]:

$$
\langle F\rangle=\sum_{i}^{n}\left(F_{i} / \sigma_{i}^{2}\right) / \sum_{i}^{n} \sigma_{i}^{-2},
$$




$$
\begin{gathered}
\langle\sigma\rangle=\left(\sum_{i}^{n}\left(1 / \sigma_{i}^{2}\right)\right)^{-0.5}, \\
\chi^{2}=\sum_{i}^{n}\left(F_{i}-\langle F\rangle\right)^{2} / \sigma_{i}^{2}, \\
\Delta F=\left((n-1)\left[\chi^{2}-(n-1)\right] / \sum_{i}^{n}\left(F_{i} / \sigma_{i}^{2}\right)\right)^{0.5}, \\
V_{\chi}=\Delta F /\langle F\rangle .
\end{gathered}
$$

Table 4 summarizes the results of the computations of these parameters. Column 2 gives the parameter $V_{\chi}$, which characterizes the relative variation amplitude, and column 3 gives the probability $p$ of variability according to the $\chi^{2}$ criterion. This parameter gives a quantitative estimate of the probability that a source whose flux densities are distributed as $\chi^{2}$ with $n-1$ degrees of freedom may be considered variable $\left(p=1-\chi^{2}(n-1)\right)$. Column 4 gives the weighted average source flux densities $\langle F\rangle$, columns 5 and 6 - the absolute $(\langle\sigma\rangle)$ and relative $\left(\left\langle\sigma_{o t n}\right\rangle\right)$ weighted average standard errors. Here $\left\langle\sigma_{\text {otn }}\right\rangle=\langle\sigma\rangle /\langle F\rangle$. Column 7 gives the variability amplitudes $\Delta F$ of the sources, columns 8 and 9the $\chi^{2}$ constant and the number $d f$ of degrees of freedom, respectively, and column 10-the mean $d H$ angles $(\overline{d H})$ averaged over all the surveys.

A comparison of the data listed in Tables 3 and 4 shows that the weighted average source flux densities $\langle F\rangle$ computed using formula (11) practically coincide with the mean values $\bar{F}$ (formula (8)) within the errors. The relative standard deviations $R M S^{\text {set }}$ exceed significantly the relative weighted standard errors $\left\langle\sigma_{o t n}\right\rangle$. The $R M S^{\text {set }}$ averaged over all the 14 sources is equal to $0.23 \pm 0.07$, and the averaged $\left\langle\sigma_{o t n}\right\rangle$ to $0.08 \pm 0.02$.

Figure $6 \mathrm{~d}$ shows $V_{\chi}$ plotted as a function of angle $d H$.

Let us now see which objects among those listed in Tables 3 and 4 can be considered variable. Kesteven et al. [39] and Fanti et al. [40] considered a source to be possibly variable if its $\chi^{2}$ probability satisfied the condition of $0.1 \% \leq 1-p \leq 1 \%$ and reliably variable if $1-p \leq 0.1 \%$.

None of the 14 sources listed in Tables 3 and 4 meet these conditions. In other words, according to the criteria of Kesteven et al. [39] and Fanti et al. [40], our sample of calibration sources contains neither variable nor likely variable objects.

Seielstad et al. [38] considered an object to be variable if $p \geq 0.985$, whereas Gorshkov and Konnikova [6] considered sources with $p \geq 0.98$ and $p \geq 0.95$ to be reliably and possibly variable, respectively. According to these criteria, the sources J $155148+045930$ and J 135137+043542 ( $p=0.96)$ may be considered to be possibly variable and the source $\mathrm{J} 103938+051031$ ( $p=$ $0.984)$ to be reliably variable. Two of these sources (J 103938+051031 and J 135137+043542) are sufficiently bright objects with flux densities $\bar{F}>100 \mathrm{mJy}$, whose transits occur at a distance of about 1.5-2 halfwidths of the vertical PBP from the central section of the survey in declination; the source $\mathrm{J} 155148+045930$ is weaker 
Table 4. The coefficient $V_{\chi}$ and the probability $p$

\begin{tabular}{|c|c|c|c|c|c|c|c|c|c|}
\hline $\begin{array}{c}R A_{2000} \quad D E C_{2000} \\
\mathrm{RCR} \\
(1)\end{array}$ & $V_{\chi}$ & (3) & $\begin{array}{r}\langle F\rangle, \\
\mathrm{mJy} \\
(4)\end{array}$ & $\begin{array}{c}\langle\sigma\rangle \\
\text { mJy } \\
(5)\end{array}$ & $\left\langle\sigma_{o t n}\right\rangle$ & $\begin{array}{c}\Delta F \\
\mathrm{mJy} \\
(7)\end{array}$ & $\chi^{2}$ & $d f$ & $\begin{array}{c}\overline{d H} \\
\operatorname{arcmin} \\
(10)\end{array}$ \\
\hline J 103938.62+051031.3 & 0.480 & 0.984 & 163 & 16 & 0.100 & 79 & 10.7 & 3 & 16.04 \\
\hline J $155148.09+045930.5$ & 0.275 & 0.961 & 74 & 7 & 0.087 & 20 & 6.9 & 2 & 3.47 \\
\hline J $135137.56+043542.0$ & 0.294 & 0.960 & 353 & 30 & 0.086 & 104 & 6.9 & 2 & -18.46 \\
\hline $\mathrm{J} 142104.21+050845.0$ & 0.280 & 0.928 & 169 & 19 & 0.110 & 48 & 5.3 & 2 & 13.69 \\
\hline J $110246.51+045916.7$ & 0.228 & 0.925 & 98 & 9 & 0.090 & 22 & 5.2 & 2 & 5.67 \\
\hline $\mathrm{J} 112437.45+045618.8$ & 0.164 & 0.895 & 446 & 24 & 0.054 & 73 & 6.1 & 3 & 2.01 \\
\hline J $101515.53+045305.6$ & 0.168 & 0.864 & 112 & 7 & 0.060 & 19 & 5.6 & 3 & -1.54 \\
\hline J $074239.34+050704.3$ & 0.180 & 0.855 & 336 & 22 & 0.066 & 60 & 5.4 & 3 & -10.75 \\
\hline J $121328.89+050009.9$ & 0.176 & 0.772 & 66 & 6 & 0.084 & 12 & 4.5 & 3 & 4.92 \\
\hline $\mathrm{J} 132448.14+0$ & 0.165 & 0.762 & 53 & 4 & 0.081 & 9 & 4.4 & 3 & 3.44 \\
\hline J $104551.72+045552.9$ & 0.083 & 0.691 & 150 & 8 & 0.055 & 12 & 3.7 & 3 & 1.43 \\
\hline J $121852.16+051449.4$ & 0.175 & 0.681 & 217 & 27 & 0.125 & 38 & 3.6 & 3 & 20.07 \\
\hline J $140730.77+044934.9$ & 0.071 & 0.674 & 78 & 8 & 0.107 & 6 & 2.2 & 2 & -5.37 \\
\hline $\mathrm{J} 134243.57+050431.5$ & 0.026 & 0.601 & 958 & 58 & 0.060 & 25 & 3.1 & 3 & 9.92 \\
\hline
\end{tabular}

$(\bar{F}=79 \mathrm{mJy})$, but passes close to the central section of the survey $\left(\overline{d H}=3.5^{\prime}\right)$.

Wang et al. [37] used the coefficient $V_{F}$ as a criterion of variability. They set its threshold value at $V_{F}=3$ and considered sources reaching this level to be variable. In other words, they considered the sources whose flux density difference $\Delta F$ in different surveys exceeds $3 \sigma$ to be variable, where $\sigma=\sqrt{\sigma_{i}^{2}+\sigma_{j}^{2}}$ (formula (2)). Of the 14 suspected variable sources in our sample only J 103938+051031 meets this condition. Another seven sources meet the condition $\Delta F>2 \sigma$.

Let us now consider the parameter $V_{\chi}$ as a variability criterion. An analysis of the data reported by Seielstad et al. [38] shows that the pa- rameters $V_{\chi}$ of variable sources (with $p \geq 0.985$ ) mostly exceed 0.2. However, there are several objects with $V_{\chi}=0.15-0.17$. Furthermore, some "non-variable" objects whose probabilities $p$ are significantly smaller than 0.985 have $V_{\chi}$ parameters greater than 0.2. In our sample of 14 sources 11 have $V_{\chi} \geq 0.164$ and only three have $V_{\chi}<0.1$.

To sum up, we can conclude that all the sources listed in Tables 3 and 4 can be considered to be possibly variable, because their flux density difference determined from the data for different observing runs exceed the sum of the flux density errors. However, the confidence level of this variability differs for different sources. Only one of them can be considered to be reliably variable 
according to the criteria of Gorshkov and Konnikova [6], Seielstad et al. [38], and Wang et al. [37], and two-possibly variable ${ }^{8}$.

The four

(J 104551+045552,

051449 , J 134243+050431, objects J $121852+$ J 140730+044934) with the lowest positive longterm variability indices $V(V=0.007-0.035)$ and $\chi^{2}$ probabilities $(0.6<p<0.7)$ are the least likely variable source candidates. Furthermore, the latter three sources change the sign of their variability indices $V$ to negative if the small systematic trend in the dependence of $G=\left(F_{i} / T_{a i}\right) /\left(A / k_{P B P}\right)$ on $d H$ in the data of the 1993 and 1994 surveys is taken into account.

The remaining seven sources (J 074239+050704, J 101515+045305, J 110246+045916, $\mathrm{J} 112437+$ 045618, J 121328+050009, J 132448+045758, and $\mathrm{J} 142104+050845)$ are intermediate between the above categories. Although they have positive long-term variability indices, their $\chi^{2}$ probabilities are low.

Thus only three out of about 80 selected calibration sources can be considered to be variable with a probability of $p>0.95$, and seven more sources can be considered to be possibly variable.

Note that eight of the 14 objects listed in Table 3 (J 074239+050704, J 103938+051031, J $110246+$

\footnotetext{
${ }^{8}$ However, even these sources cannot be considered variable according to the more stringent statistical criteria adopted by Kesteven et al. [39] and Fanti et al. [40].
}

045916, J 121328+050009, J 132448+045758, $\mathrm{J} 135137+043542, \quad \mathrm{~J} 142104+050845, \quad$ and J $155148+$

045930) have variability indices $V \gtrsim 0.08$, which are comparable with the long-term variability indices determined by Afanas'ev et al. [41] for variable sources with flat spectra.

Let us now present the more detailed properties of all of the 14 candidate objects, since they all have optical identifications.

The object J $074239+050704$ (or $4 \mathrm{C}+05.33$ ) was identified with a galaxy and its color indices according to the data of the WISE mid-infrared survey [42] are typical of a spiral galaxy. The NED database confirms this conclusion: the object is classified as a Seyfert type galaxy (Sy2) with $Z=0.16$. According to the GSC [43] and USNO-B1 [44] catalogs, the scatter of the magnitudes of the object in close filters amounts to 1. 2 , and this fact may be indicative of optical variability. This source was studied by Gorshkov and Konnikova [6].

The object J 101515+045305 (PMN J 1015+0452) is a point source. On the FIRST maps, it was identified with a galaxy, which by its color index $(u-r)>2.22$ [45] can be classified as an early-type galaxy.

The object $\quad J 103939+051031$ (or PKS $\mathrm{J} 1037+05$ ) is a binary source (FIRST) with the so-called "winged" morphology, which may be indicative of the interaction of the plasma back flow from the lobes of the radio source with 
the nonuniform environment. Another model explains such a morphology by "ageing" components that remained from fast reorientation of the black hole and accretion disk as a result of merging and subsequently resumed nuclear activity $[46,47]$. The object has been identified with an optically variable elliptical galaxy $(Z=0.068)$ of the Abell 1066 cluster located close to two other galaxies. It is the most likely candidate variable object according to the variability criteria employed.

The J 110246+045916 object has the same morphology as J $103939+051031$ and is identified with a possibly optically variable starlike object, supposedly a quasar.

The $\mathrm{J} 112437+045618(4 \mathrm{C}+05.50)$ object is a double source from the sample of the RC catalog objects with steep spectra. Its optical identification and spectrum were obtained on the 6-m telescope of the Special Astrophysical Observatory of the Russian Academy of Sciences within the framework of the "Big Trio" program of the search for distant galaxies [48]. Parijskij et al. [49] classified the optical object as a galaxy $(Z=0.284)$ with narrow emission and absorption lines in its spectrum. In the SDSS survey [50] the object is classified as a Seyfert galaxy (Sy2) with $Z=0.283$, possibly optically variable.

The J 121328+050009 (PMN J 1213+0500) object is a double source with a nucleus, which, like $\mathrm{J} 112437+045618$, is a part of the SS sample of the RC catalog; it was identified with a galaxy $\left(Z_{p h}=0.76\right)$.
The J132448+045758 object is a double source identified with a starlike object, probably a quasar.

The J 135137+043542 (MRC J 1349+048) object is a point source, which was studied by Gorshkov and Konnikova [6]. It is identified in the SDSS with a faint galaxy. We consider it to be a variable radio source.

The $\mathrm{J} 142104+050845$ object is a point source identified with a galaxy $(Z=0.455)$, which may be a part of a triplet. It is possibly an optically variable object.

The J 155148+045930 (PMN J 1551+0458) object is a double source; it is, like $\mathrm{J} 112437+045618$ and $\mathrm{J} 121328+050009, \quad \mathrm{a}$ part of the SS sample of the RC catalog. The object is identified with a faint $R=23^{\mathrm{m}}$. 6 galaxy. We consider it to be a variable radio source.

We selected the next four sources as candidate variable objects, but they failed to meet the adopted variability criteria and, according to the available data, we cannot classify them as variable.

The J 104551+045553 (PMN J 1045+0455) object is a likely double source identified with a galaxy in the SDSS survey.

The J $121852+051449$ object is a double source identified with an optically variable elliptical galaxy $(Z=0.078)$, which is the brightest in the Abell 1516 cluster.

The J $134243+050431(4 \mathrm{C}+05.57)$ object is an FRI-type double source identified with an op- 
tically variable Seyfert galaxy (Sy1), $Z=0.136$.

The J140730+044934 object is a double source identified with a quasar candidate with $Z_{p h}=1.775$, which is possibly optically variable.

Nine out of 14 sources listed in Table 3 show optical variability: J 074239+050704, J 103938+051031, J 110246+045916， J 112437+045618，J 121328+ 050009， J 121852+051449， J 134243+050431， $\mathrm{J} 140730+044934$, and $\mathrm{J} 142104+050845$. The data for the remaining sources is too scarce to allow any conclusions.

Figures 10-13 show the light curves (left panels) and spectra (right panels) of the calibration sources from Tables 3 and 4: Fig. 10 shows the light curves and spectra for objects with $p>$ 0.95, Fig. 11-for objects with $0.89<p<0.95$, Fig. 12-for objects with $0.75<p<0.89$, and Fig. 13-for objects with $0.6<p<0.7$.

Figures 14 and 15 show, for comparison, the light curves (left panel) and spectra (right panel) of "nonvariable" sources with long-term variability indices $V<0$.

\section{CONCLUSIONS}

To find variable sources in the data of the deep surveys carried out on the RATAN-600 radio telescope in 1980-1994, we performed a more thorough selection of calibration sources, constructed the experimental dependences $F / T_{a}$ and the computed calibration curves, performed a detailed analysis and estimated the relative stan- dard errors for each survey.

To test the calibration sources for variability, we performed quantitative estimates of the parameters that characterize the variability of objects (our main parameter was the long-term variability index $V$ ) and analyzed the statistical properties of suspected variable objects.

Out of the entire sample of calibration sources (about 80 objects) 14 had positive long-term variability indices for at least one pair of surveys. Eight of these sources have the long-term variability indices $V>0.08$ and for 10 sources the maximum flux densities exceed their minimum flux densities by more than a factor of 1.5.

We estimated the $\chi^{2}$ variability probabilities $p$ and the parameter $V_{\chi}$ that characterizes the relative amplitude of variability for these 14 sources.

We found three objects to be the most likely variable source candidates: J 155148+045930 $(p=0.961), \mathrm{J} 135137+043542(p=0.960)$, and $\mathrm{J} 103938+051031(p=0.984)$.

The J 103938+051031 source also meets the variability condition according to the $V_{F}>3 \sigma$ criterion, where $\sigma=\sqrt{\sigma_{i}^{2}+\sigma_{j}^{2}}\left(\sigma_{i}\right.$ and $\sigma_{j}$ are the root mean square errors of the source flux density in the $i$-th and $j$-th surveys). Seven sources have $2 \sigma<V_{F}<3 \sigma$, and the probability of variability $p\left(\chi^{2}\right)>0.85$. The remaining six objects of this sample have $0.6<p<0.8$.

Note, however, that two of the three most likely candidate variable sources, $\mathrm{J} 103938+051031$ and $\mathrm{J} 135137+043542$, in 

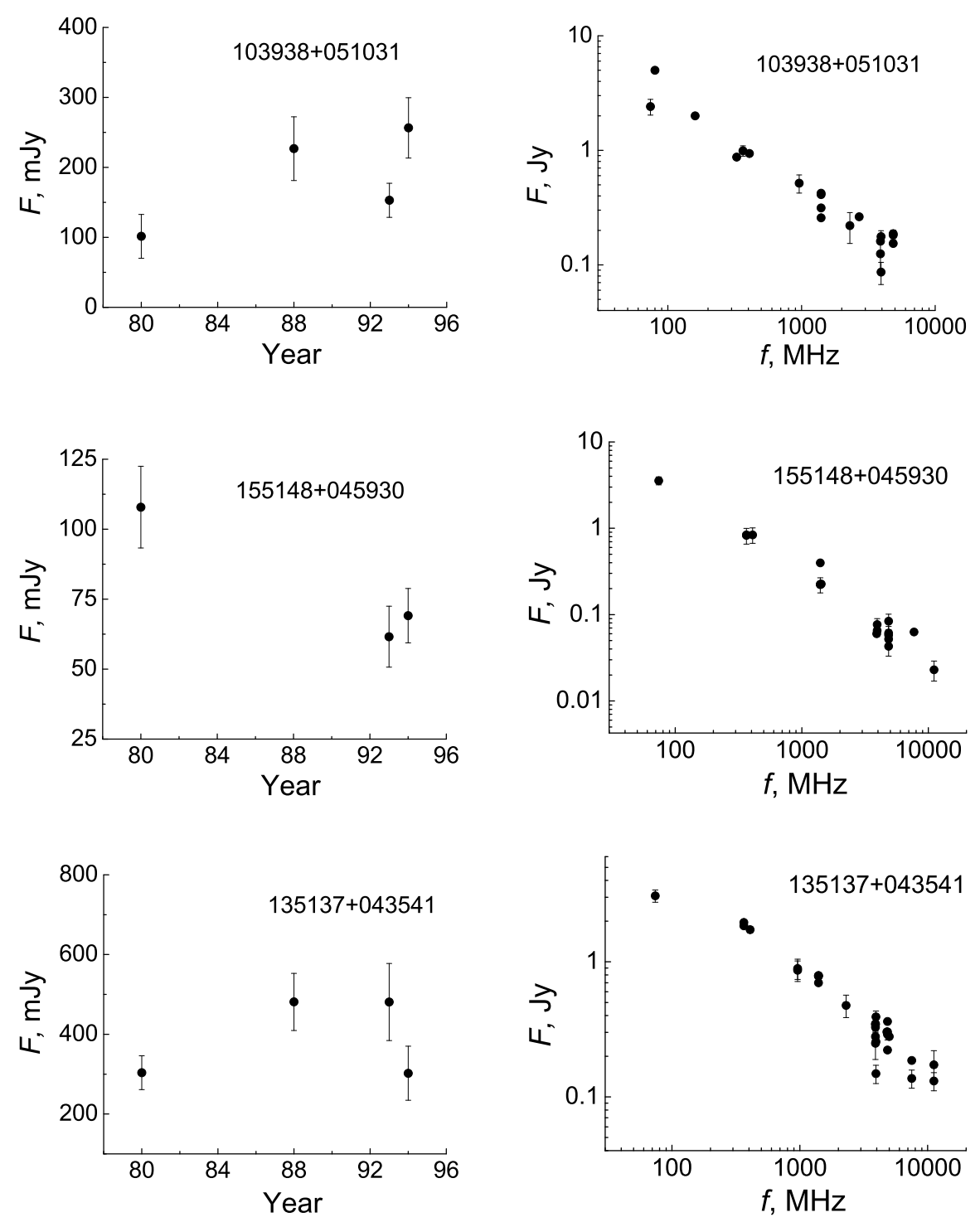

Figure 10. Light curves (left) and spectra (right) of suspected variable calibration sources $(V>0)$ with the probabilities $p\left(\chi^{2}\right)>0.95$.

one of the surveys pass rather far from its by Kesteven et al. [39] and Fanti et al. [40], central section $\left(|d H|>18^{\prime}\right)$. Although they namely that only the sources with $p>0.99$ and show up conspicuously in the records, their flux $p>0.999$ can be considered variable and reliably densities are more difficult to determine than variable, respectively, then there are no variable those of the sources that are close to the central sources in our sample.

section, primarily because they are extended features.

Nine out of 14 sources show a scatter of magnitudes in close filters ranging from 0.8 If we employ the more stringent criteria used to $3^{\mathrm{m}}$ according to the data of the GSC and 

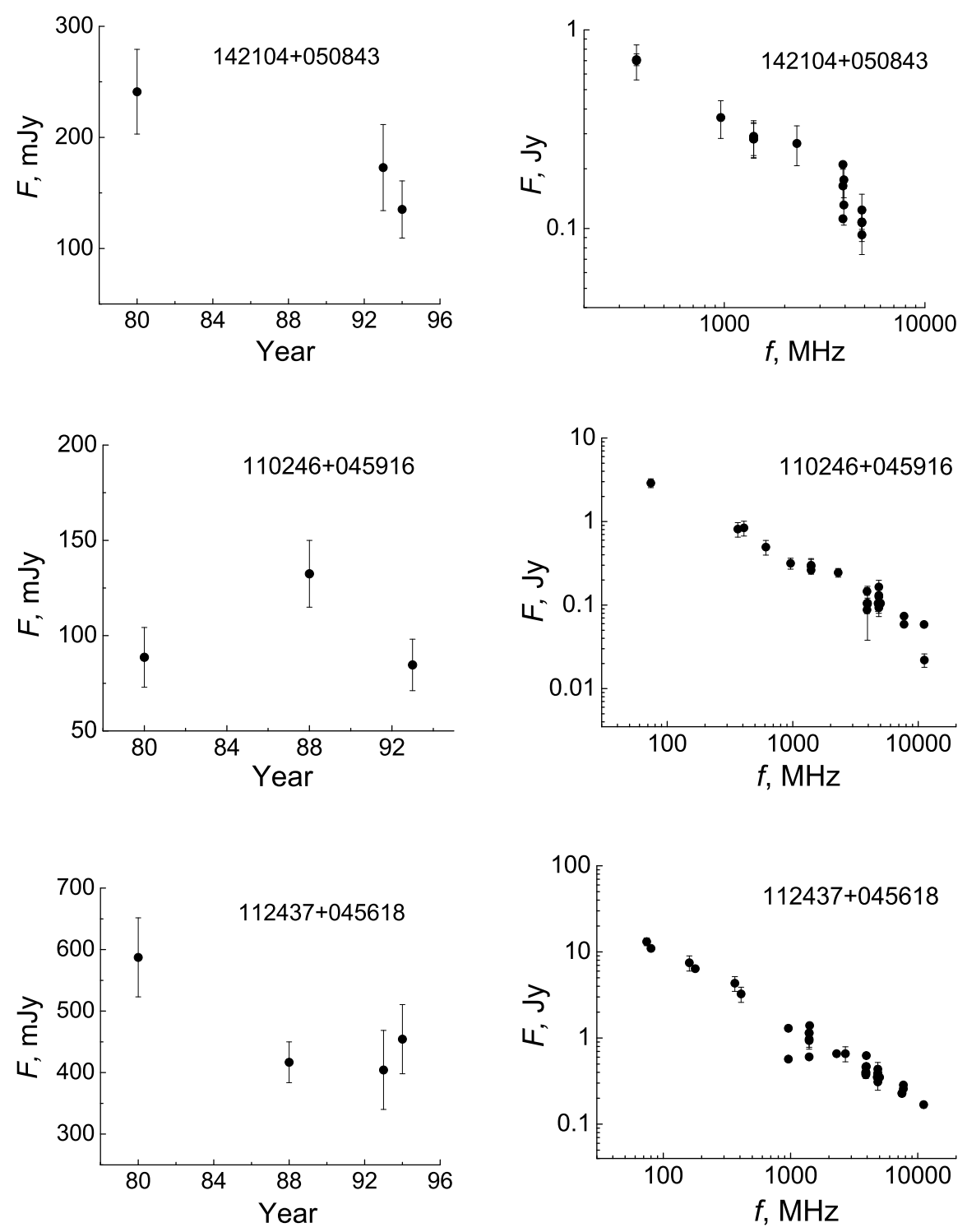

Figure 11. Same as Fig. 10 for the sources with $0.89<p\left(\chi^{2}\right)<0.95$.

USNO-B1 catalogs and the 2MASS, SDSS, and LAS UKIDSS surveys, which is indicative of optical variability.

The estimates of relative standard deviations of flux densities from their mean values averaged over all the surveys, $R M S^{\text {set }}$, for the subsamples with $V>0$ and $V<0$ showed that they differ significantly. The $R M S^{\text {set }}$ val- ues for suspected variable sources and for "nonvariable" sources, averaged over the entire sample, are equal to $R M S^{\text {set }}=0.23 \pm 0.07$ and $R M S^{\text {set }}=0.08 \pm 0.04$, respectively. This leads us to conclude that the flux densities of the overwhelming majority of calibration sources varied only slightly from one survey to another, and that the flux density errors, on average, did not 

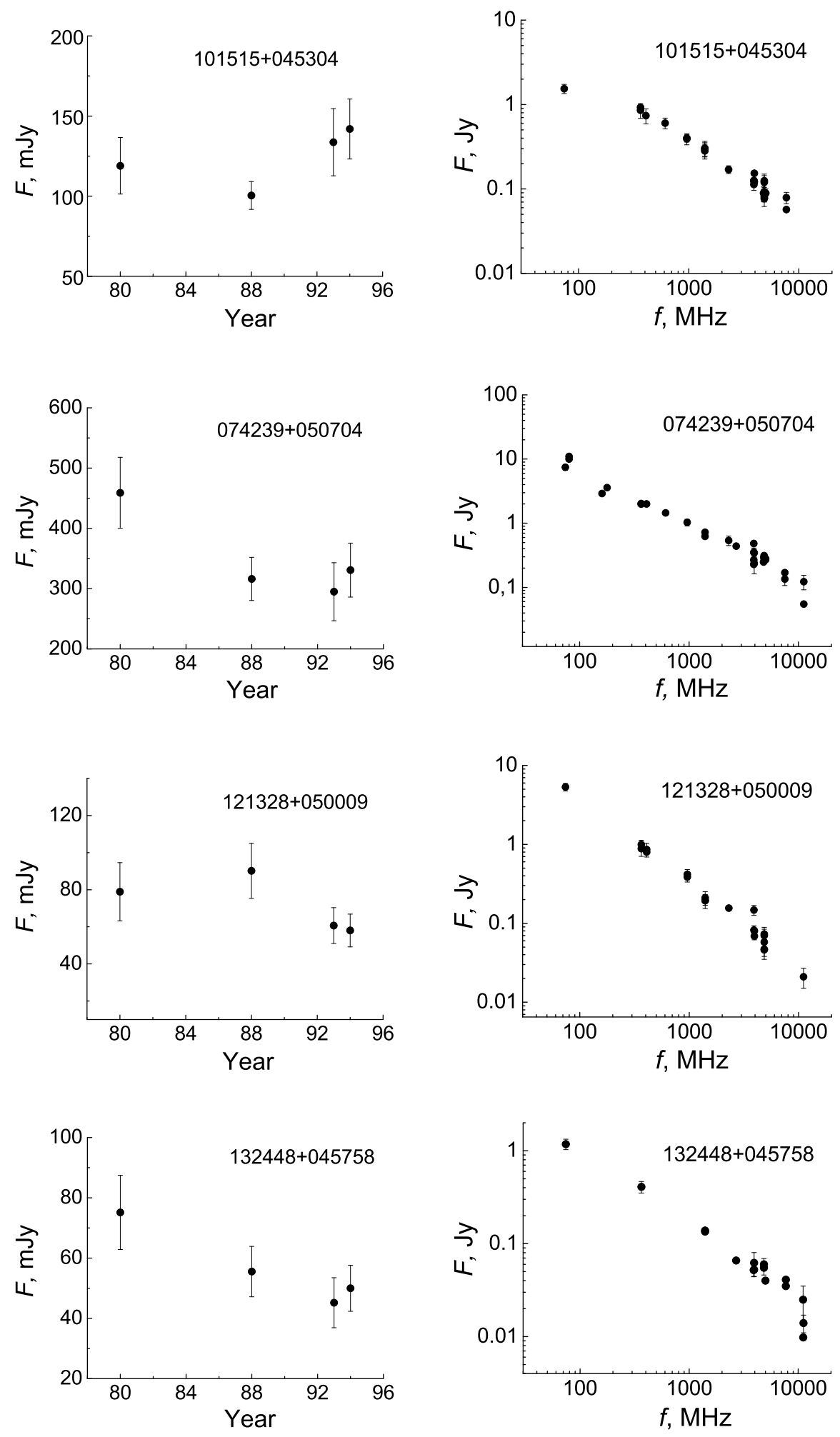

Figure 12. Same as Fig. 10 for the sources with $0.75<p\left(\chi^{2}\right)<0.89$. 

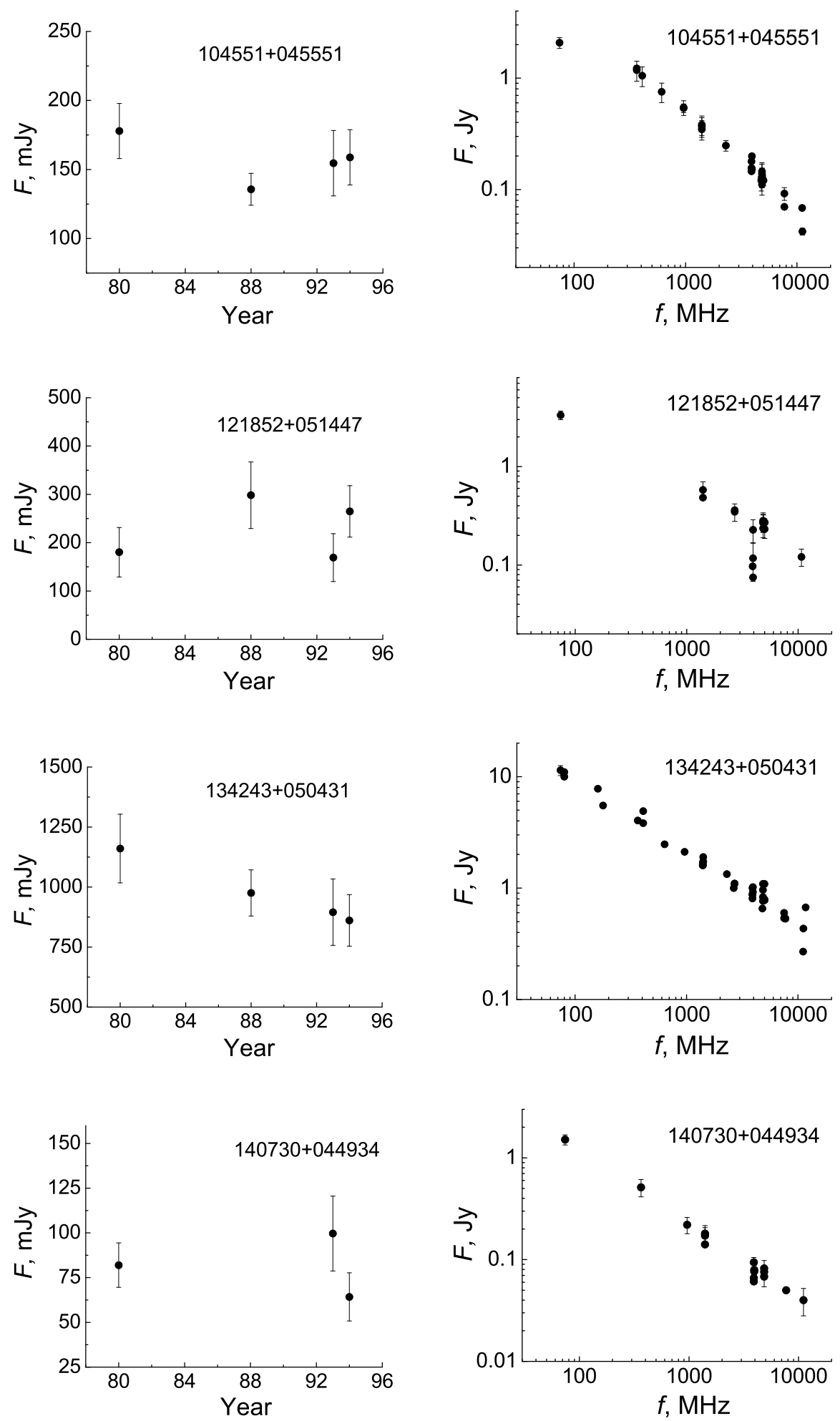

Figure 13. Same as Fig. 10 for the sources with $0.6<p\left(\chi^{2}\right)<0.7$. 

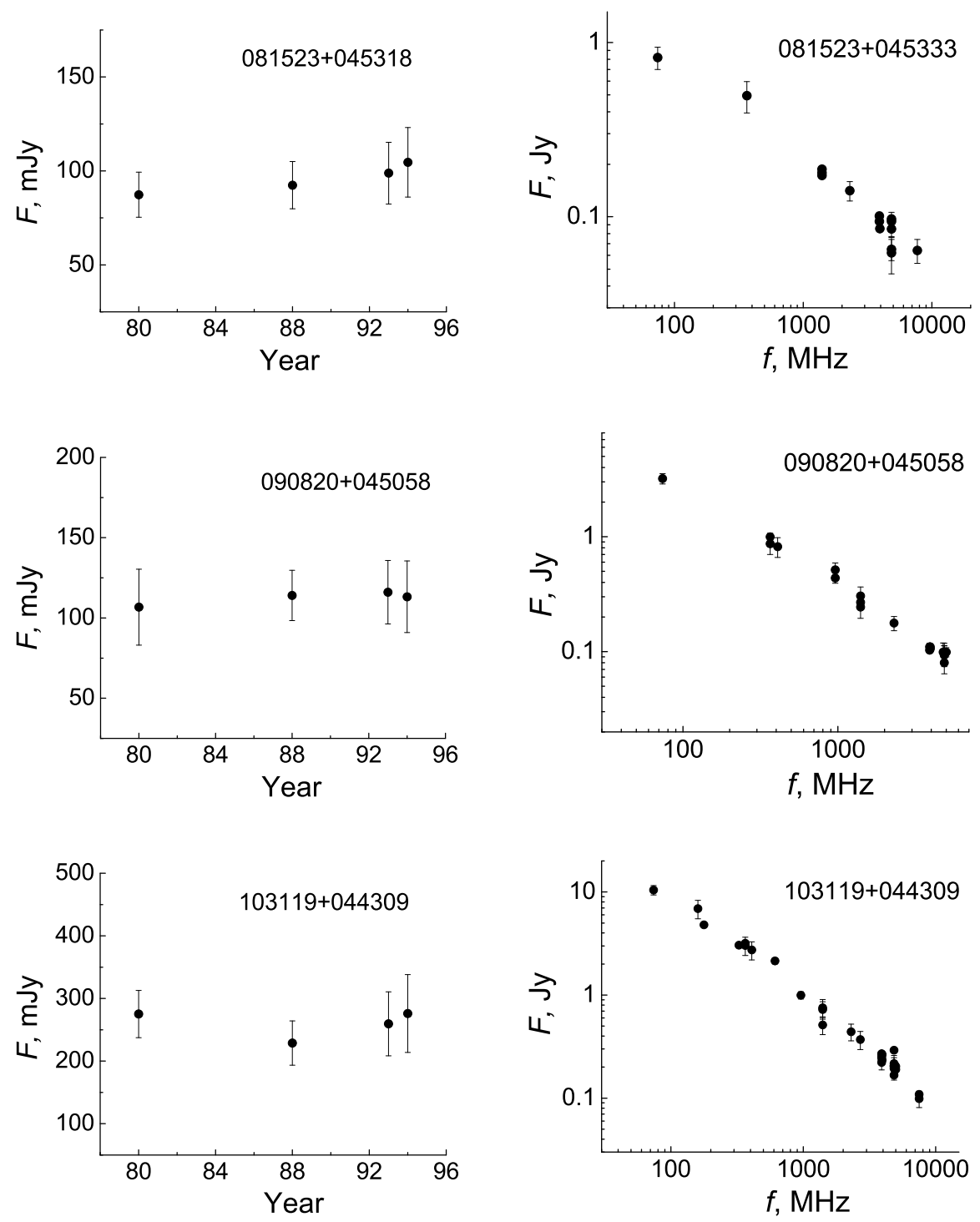

Figure 14. Same as Fig. 10, but for "non-variable" objects with long-term variability indices $V<0$.

exceed $10 \%$.

\section{ACKNOWLEDGMENTS}

This study is our tribute and gratitude to

The calibrating curves and estimates of the Natalya Sergeevna Soboleva, her longstanding relative standard errors of the inferred flux den- and fundamental work on deep search surveys sities obtained in this study will make it possi- performed on the RATAN-600 radio telescope. ble to search for variable sources among a bigger This work was supported in part by the Russian sample of objects observed in different surveys, Foundation for Basic Research (grants nos. 11as we plan to do in our forthcoming papers. $\quad 02-12036,11-02-00489$ and 10-07-00412) and the 

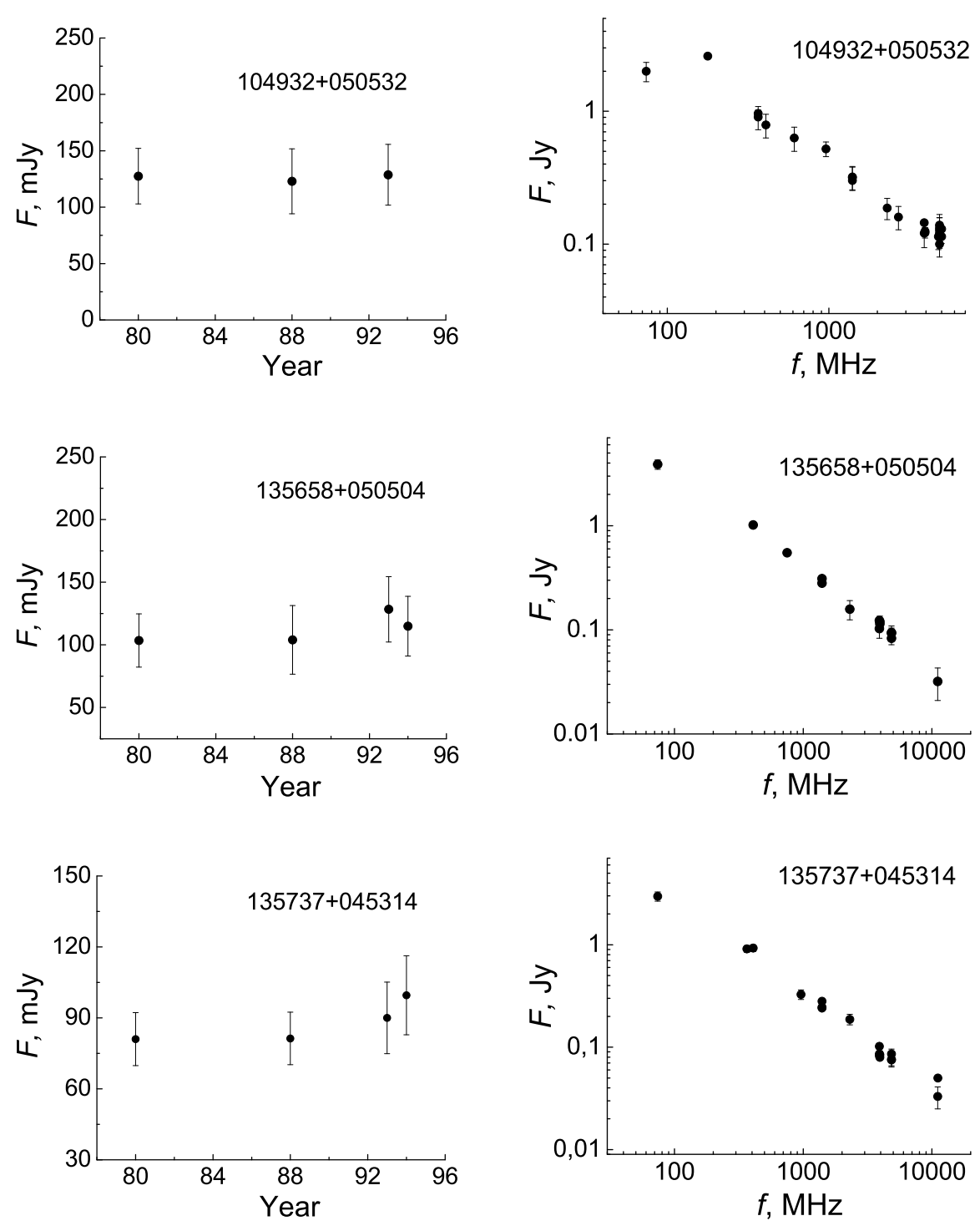

Figure 15. Same as Fig. 14.

Ministry of Education and Science of the Russian France, as well as the NASA/IPAC Extragalactic Federation (state contracts nos. 16.552.11.7028 Database (NED), operated by the Jet Propulsion and 16.518.11.7062). In this research we used Laboratory, California Institute of Technology, the VizieR catalogue access tool and the SIM- under the contract with the National AeronauBAD database, operated at CDS, Strasbourg, tics and Space Administration.

1. Yu. N. Pariiskii and D. V. Korol'kov, Itogi Nauki Tekh., Ser.: Astron. 31, 73 (1986).

2. V. R. Amirkhanyan, A. G. Gorshkov, A. A. Ka- pustkin, et al., Soobshch. Spets. Astrofiz. Obs. 47, 5 (1985).

3. Catalog of Radio Sources of the Zelenchuk Sky 
Survey in the $0^{\circ}-14^{\circ}$ Declination Range, Ed. by

M. G. Larionov (Moscow University, 1989) [in Russian].

4. V. R. Amirkhanyan, A. G. Gorshkov, and V. K. Konnikova, Sov. Astron. Lett. 15, 378 (1989).

5. V. R. Amirkhanyan, A. G. Gorshkov, and V. K. Konnikova, Sov. Astron. 36, 115 (1992).

6. A. G. Gorshkov and V. K. Konnikova, Astron. Rep. 39, 257 (1995).

7. Yu. A. Kovalev, Soobshch. Spets. Astrofiz. Obs. 68, 60 (1991).

8. Yu. A. Kovalev, Bull. Spec. Astrophys. Obs. 44, 50 (1997).

9. A. G. Gorshkov, V. K. Konnikova, and M. G. Mingaliev, Astron. Rep. 44, 161 (2000).

10. A. G. Gorshkov, V. K. Konnikova, and M. G. Mingaliev, Astron. Rep. 47, 903 (2003).

11. A. G. Gorshkov, V. K. Konnikova, and M. G. Mingaliev, Astron. Rep. 52, 278 (2008).

12. Yu. V. Sotnikova, M. G. Larionov, and M. G. Mingaliev, Astrophysical Bulletin 64, 185 (2009).

13. A. G. Gorshkov, V. K. Konnikova, and M. G. Mingaliev, Astron. Rep. 54, 908 (2010).

14. Yu. N. Parijskij and D. V. Korolkov, Sov. Sci. Rev. Astrophys. Space Phys. 5, 39 (1986).

15. N. N. Bursov, Yu. N. Pariiskii, E. K. Majorova, et al., Astron. Rep. 51, 197, (2007).

16. N. N. Bursov, Candidate's Dissertation in Mathematics and Physics (Special Astrophysical Observatory, Nizhnii Arkhyz, 2003).

17. Yu. N. Parijskij, N. N. Bursov, N. M. Lipovka, et al., A\&AS 87, 1 (1991).

18. Yu. N. Parijskij, N. N. Bursov, N. M. Lipovka, et al., A\&AS 96, 583 (1992).

19. N. N. Bursov, Astron. Rep. 41, 35 (1997).
20. N. S. Soboleva, N. N. Bursov, A. V. Temirova, Astron. Rep. 50, 341 (2006).

21. N. S. Soboleva, E. K. Majorova, O. P. Zhelenkova, et al., Astrophysical Bulletin 65, 42 (2010).

22. S. P. Spangler and D. B. Cook, AJ 85, 659 (1980).

23. M. F. Aller, H. D. Aller, and P. H. Hughes, AJ 586, 33 (2003).

24. J. J. Condon, W. D. Cotton, E. W. Greisen, et al., AJ 115, 1693 (1998).

25. NED, http://nedwww.ipac.caltech.edu/.

26. A. S. Cohen, W. M. Lane, W. D. Cotton, N. E. Kassim, et al., AJ 134, 1245 (2007).

27. P. C. Gregory, W. K. Scott, K. Douglas, and J. J. Condon, ApJS 103, 427 (1996).

28. N. A. Esepkina, N. L. Kaidanovskii, B. V. Kuznetsov, et al., Radiotekhnika i Elektronika 6, 1947 (1961).

29. N. A. Esepkina, N. S. Bakhvalov, B. A. Vasil'ev, et al. Astrofiz. Issled. (Izv. Spets. Astrofiz. Obs. ) 11, 182 (1979).

30. N. A. Esepkina, B. A. Vasil'ev, I. A. Vodovatov, and M. G. Vysotskii, Astrofiz. Issled. (Izv. Spets. Astrofiz. Obs. ) 11, 197 (1979).

31. E. K. Majorova, Bull. Spec. Astrophys. Obs. 53, 78 (2002).

32. E. K. Majorova and S. A. Trushkin, Bull. Spec. Astrophys. Obs. 54, 89 (2002).

33. O. V. Verkhodanov, B. L. Erukhimov, M. L. Monosov, et al., Preprint of the Special Astrophysical Observatory of the Russian Academy of Sciences, 78, 1 (1992).

34. E. K. Majorova and N. N. Bursov, Astrophysical Bulletin 62, 378 (2007).

35. E. K. Majorova, Astrophysical Bulletin 65, 196 (2010). 
36. W. H. de Vries, R. H. Becker, R. L. White, and D. J. Helfand, AJ 127, 2565 (2004).

37. Ting-Gui Wang, Hong-Yan Zhou, JungXian Wang, et al., Astrophys. J. 645, 856 (2006).

38. G. A. Seielstad, T. J. Pearson, and A. C. S. Readhead, PASP 95, 842 (1983).

39. M. J. L. Kesteven, A. H. Bridle, and G. W. Brandie, AJ 81, 919 (1976).

40. R. Fanti, A. Ficarra, F. Mantovani, and L. Padrielli, A\&AS 36, 359 (1979).

41. V. L. Afanas'ev, S. N. Dodonov, A. V. Moiseev, et al., Astron. Rep. 53, 287 (2009).

42. E. L. Wright, P. R. M. Eisenhardt, A. K. Mainzer, et al., AJ 140, 1868 (2010).

43. B. M. Lasker, M. G. Lattanzi, B. J. McLean, et al., AJ 136, 735 (2008).

44. D. G. Monet, S. E. Levine, B. Canzian, et al., AJ 125, 984 (2003).

45. I. Strateva, Z. Ivezić, G. R. Knapp, et al., AJ 122, 1861 (2001).

46. C. C. Cheung, AJ 133, 2097 (2007).

47. D. Cseh, S. Frey, Z. Paragi, et al., A\&A 523, 34 (2010).

48. Yu. N. Parijskij, N. S. Soboleva, W. M. Goss, et al., IAUS 175, 591 (1996).

49. Yu. N. Parijskij, A. I. Kopylov, A. V. Temirova, et al., Astron. Rep. 54, 675 (2010).

50. K. Abazajian, J. K. Adelman-McCarthy, M. A. Agüeros, et al., ApJS 182, 543 (2009). 